\title{
Enhanced Co-Primary Spectrum Sharing Method for Multi-Operator Networks
}

\author{
Petri Luoto ${ }^{\circledR}$, Student Member, IEEE, Mehdi Bennis, Senior Member, IEEE, \\ Pekka Pirinen, Senior Member, IEEE, Sumudu Samarakoon, Student Member, IEEE, \\ and Matti Latva-Aho, Senior Member, IEEE
}

\begin{abstract}
We consider a multi-operator small cell network where mobile network operators are sharing a common pool of radio resources. The goal is to ensure long term fairness of spectrum sharing without coordination among small cell base stations. It is assumed that spectral allocation of the small cells is orthogonal to the macro network layer, and thus, only the small cell traffic is modeled. We develop a decentralized control mechanism for base stations using the Gibbs sampling based learning technique, which allocates a suitable amount of spectrum for each base station. Five algorithms are compared addressing co-primary multi-operator resource sharing under heterogeneous traffic requirements and the performance is assessed through extensive system-level simulations. The main performance metrics are user throughput and fairness between operators. The numerical results demonstrate that the proposed Gibbs sampling based learning algorithm provides about tenfold cell edge throughput gains compared to state-of-the-art algorithms, while ensuring fairness between operators.
\end{abstract}

Index Terms-Fairness, heterogeneous traffic requirements, resource allocation, small cell networks, system level simulations

\section{INTRODUCTION}

$\mathrm{M}$ $\mathrm{UCH}$ more spectrum will be needed to meet the increasing traffic demand of future wireless systems. Radio frequencies above $6 \mathrm{GHz}$ have the potential to allow wider bandwidths and thus, provide enough capacity for the 2020-2030 time frame. Depending on the carrier frequency, spectrum needs will include large chunks of spectrum in high frequency bands and flexible use of spectrum through advanced spectrum sharing techniques [1].

Mobile network operators (MNOs) typically acquire exclusive usage rights for certain frequency bands and have little incentives to share spectrum with other operators. However, due to higher cost and spectrum scarcity it is expected that an efficient use of spectrum in the fifth generation (5G) networks will rather rely on spectrum sharing than exclusive licenses, especially when higher carrier frequencies are considered [2].

One of the advanced spectrum sharing techniques is coprimary spectrum sharing (CoPSS), which is proposed as a sharing mechanism towards $5 \mathrm{G}$ systems, where any $\mathrm{MNO}$ is allowed to utilize the shared spectrum allocated for $5 \mathrm{G}$ cellular systems [2]. In [3], CoPSS is defined as a spectrum access model where primary license holders agree on the joint use of (or parts of) their licensed spectrum. This is only possible in the small cell domain where base stations' coverage is not broad and the frequency band is dedicated to small cell use.

- The authors are with the Centre for Wireless Communications, University of Oulu, Oulu 90014, Finland. E-mail: \{petri.luoto, mehdi.bennis, pekka. pirinen, sumudu.samarakoon, matti.latva-aho\}@ee.oulu.fi.
Multi-operator spectrum sharing has been considered in many studies over the years [4], [5], [6], [7]. In [8], various aspects of inter-operator resource sharing, such as analyzing efficient coordination mechanisms and developing frameworks for infrastructure sharing, have been studied. In [9], the potential gain of spectrum sharing between cellular operators is investigated in terms of network efficiency. In [10], inter-operator sharing of cellular resources including capacity, spectrum and base stations is investigated. Most of the multi-operator spectrum sharing research has been done in macro cell networks.

In [11], enabling/limiting factors for CoPSS are discussed. Therein, the current scarcity of spectrum and new business potential, especially in hotspots and small cells, are seen as enabling factors for CoPSS. The limited availability of suitable spectrum for sharing, a low level of technical/ business knowledge among MNOs regarding CoPSS, and lack of rules to coordinate sharing between MNOs with similar customer profiles, are seen as limiting factors. The findings suggest that substantial further research is required, not only from a technical perspective, but also from a business perspective.

A CoPSS method for multiple operator networks, in dense local area deployment is considered in [12]. A realistic system framework is formulated to guarantee reliable and efficient communications within a denser network. The proposed solution is easy to control and incurs low signaling overhead. In [13], a CoPSS scenario where operators having similar rights for accessing spectrum is evaluated. A coordination protocol is designed which assumes an agreement to a set of negotiation rules. Signaling overhead is low and other MNOs' channel state information is assumed to be unknown. In [14], CoPSS with multi-user MIMO in two small cell networks is 
considered. Each base station allocates its users over the shared band, when the number of subcarriers in the dedicated band is not enough to serve all users. Subcarrier and power allocation is proposed based on this scenario.

One way to enable CoPSS is to leverage software-defined architecture based on novel ideas from network function cloudification and network virtualization as proposed in [15]. Furthermore, in [16] QoS-aware virtualization and routing is proposed in software defined networks (SDN). The proposed solution is shown to be effective for practical SDN implementations, which allows resource sharing among network operators.

In [17] we mainly focussed on the possible gains in the achieved throughput when MNOs have similar traffic patterns and spectrum sharing is done at the physical resource block (PRB) level. In [18] we introduced a decentralized algorithm where different MNOs are sharing a common pool of component carriers (CCs) and MNOs have different traffic requirements. Here, we extend these previous works by introducing a new decentralized spectrum sharing algorithm, showing its convergence and rigorous system level simulation analysis.

In previous works [13], [14], and [17], there is always coordination between MNOs. However, the amount of coordination among MNOs should be minimal because MNOs may not be willing to share operator-specific information with competitors. Thus, information exchange among MNOs is a critical design factor for spectrum sharing algorithms. Furthermore, in [14] and [17] the spectrum is shared at subcarrier or PRB level. This is challenging because different MNOs' SCNs need to be synchronized. However, this type of spectrum sharing guarantees more efficient utilization of the spectrum. When multi-operator networks are not jointly synchronized the more practical approach is to share spectrum at coarser granularity, e.g., at component carrier level, which is considered in this work. The goal is to ensure the long term fairness by minimizing a time average cost function per SBS, without coordination among small cell base stations. A dense indoor network deployment, consisting of multiple small cell base stations (SBSs) per building operated by three independent MNOs, is considered. Traffic in the network is continuous with constant rate. To solve the per SBS cost minimization problem in a decentralized manner with no SBS coordination, we propose CoPSS algorithms using a learning tool based on Gibbs sampling [19], which allocates suitable amount of spectrum for each base station while avoiding interference among SBSs and maximizing the total network throughput. The proposed algorithms are compared with a number of state-of-the-art baselines.

The core of the extensive LTE-A system level network simulator has been built according to the International Telecommunication Union's system level simulation guidelines [20] and calibrated and rigorously evaluated in selected macro and microcell environments [21], [22]. The simulator is extended to incorporate indoor femtocells, calibrated and verified in [23] and previously utilized in [24], [25].

The contributions of this paper can be summarized as follows:

(1) We propose a new decentralized spectrum sharing algorithm using a learning tool based on Gibbs

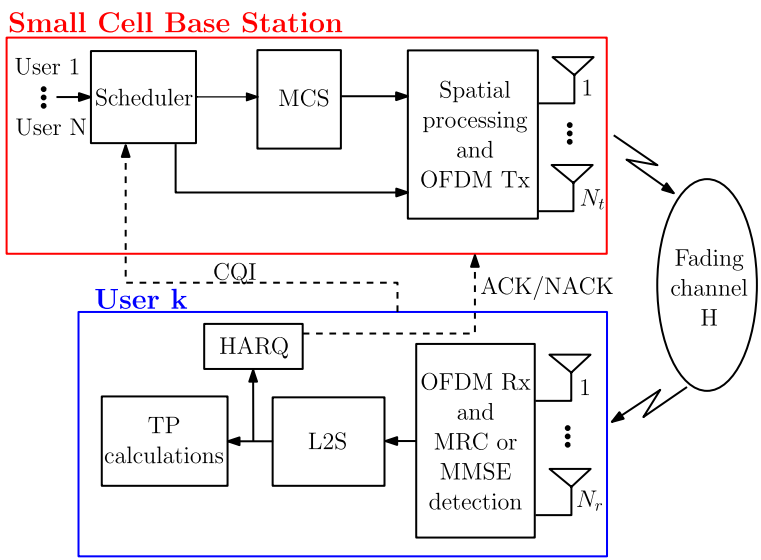

Fig. 1. Block diagram of the link model.

sampling. Moreover, a novel idea is adopted to enhance the speed of the learning process.

(2) We analyze the convergence and optimality of the proposed algorithm.

(3) Effectiveness of the proposed spectrum sharing algorithms are validated by comparing them with three state-of-the-art models.

(4) We utilize comprehensive system level simulations in the analysis, where a dense indoor network deployment, consisting of multiple SBSs per building operated by three independent MNOs, is considered.

(5) Based on the simulation results we conclude the applicability of our methods to different scenarios.

This paper is organized as follows. The system and link models are defined in Section 2. In Section 3, the actual CoPSS algorithms are elaborated and the performance metrics are introduced. Section 4 provides the convergence analysis and the performance comparison for the proposed CoPSS algorithms. Finally, Section 5 concludes the paper.

\section{LINK AND SySTEM MOdELS}

Consider the downlink of an Orthogonal Frequency-Division Multiple Access (OFDMA) small cell base station (SBS) network where a set of SBSs $\mathcal{B}$, operated by a set of MNOs $\mathcal{L}=\{1, \ldots, l\}$, are deployed. MNO $l$ controls a set of its own SBSs $\mathcal{B}_{l}$, and thus $\mathcal{B}=\cup_{\forall l \in \mathcal{L}} \mathcal{B}_{l}$ with $\mathcal{B}_{l} \cap \mathcal{B}_{l^{\prime}}=\emptyset$ for all $l \neq l^{\prime}$. Each SBS $b \in \mathcal{B}$ has $K$ users. The frequency domain resource consists of $N_{\mathrm{c}}$ subcarriers, where 12 subcarriers form a physical resource block and 6 PRBs form a component carrier (CC). A common pool consists of $N_{\text {cc }}$ number of CCs. It is assumed that the spectral allocations of the SBSs are orthogonal to the macrocell network layer, and thus only the small cell traffic is modeled. Moreover, we assume that SBSs do not coordinate with each other.

The link model between a SBS and a user is illustrated in Fig. 1. A link-to-system (L2S) interface is used in the simulations. Each user is paired to the SBS based on the path loss model [26]. A geometry-based stochastic channel model (GSCM) [27] is used to model fast fading and shadowing losses for all links. Channel parameters are determined stochastically, based on the statistical distributions extracted from channel measurements. SBS related assumptions for the links are adopted from [26]: 

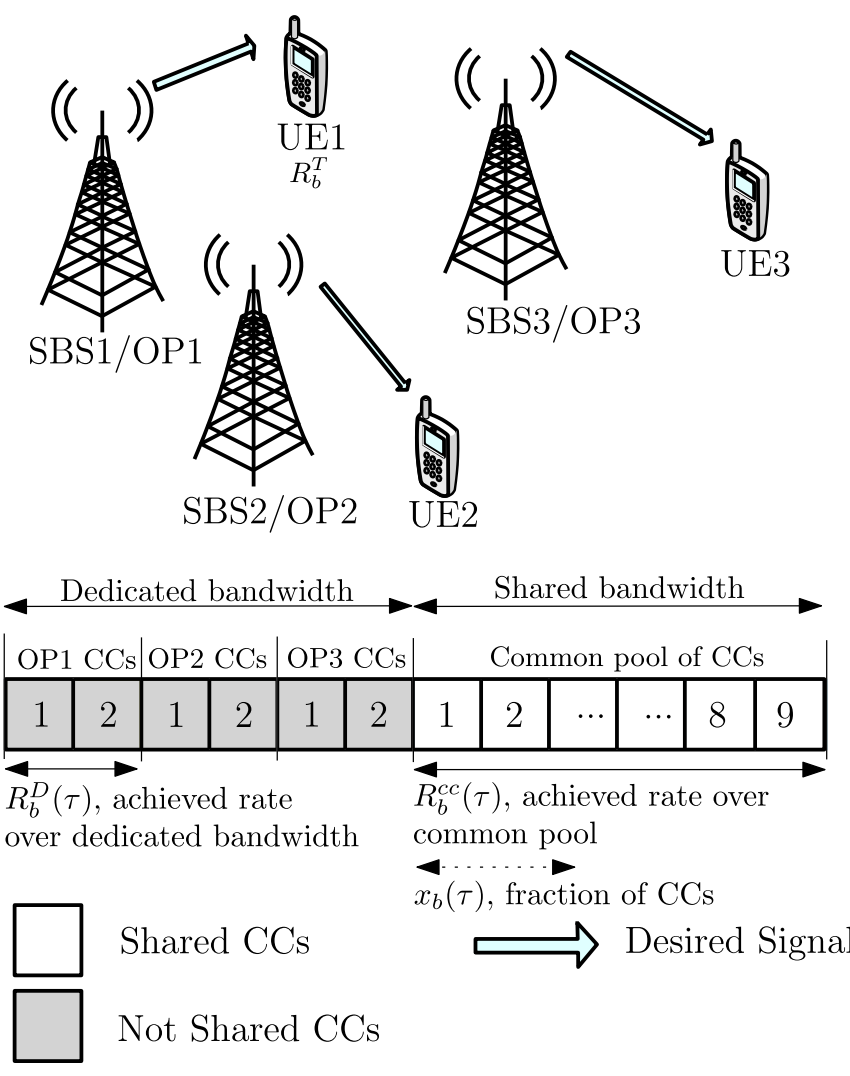

Fig. 2. System model and bandwidth allocation.

all links are assumed to be non-line-of-sight (NLOS) and users are always inside buildings.

User $k$ estimates channel-quality indicator (CQI) information, i.e., quantized signal-to-interference-plus-noise ratio (SINR), from the received signal for every CC and send it to the SBS. The uplink channel is assumed to be ideal, i.e., error free. In order to model a practical closed loop system, periodic and delayed CQI is assumed. At SBS, the proportional fair scheduler utilizes the CQI information in the allocation of frequency resources to the most suitable users. After scheduling, the link adaptation is performed for scheduled users by selecting modulation and coding scheme (MCS) based on CQI information. Finally, the data is sent over the fading channel. The cyclic prefix is assumed to be longer than the multipath delay spread, and thus inter-symbol-interference is not considered.

At the receiver, perfect frequency and time synchronization is assumed. Link-to-system mapping is performed using mutual information effective SINR mapping (MIESM) [28]. This significantly reduces the computational overhead in comparison to the exact modeling of the radio links, while still providing sufficiently accurate results. In the L2S interface, SINR is calculated and mapped to corresponding average mutual information. Based on the MIESM value, the frame error probability (FEP) is approximated according to a predefined frame error rate (FER) curve of the used MCS. Based on the FER, successful and erroneous frames can be detected, and hybrid automatic repeat request (HARQ) can take the control of retransmissions. An acknowledgement (ACK) or a negative acknowledgement (NACK) message is sent back to the SBS to signal the success or failure of the transmission, respectively. The results

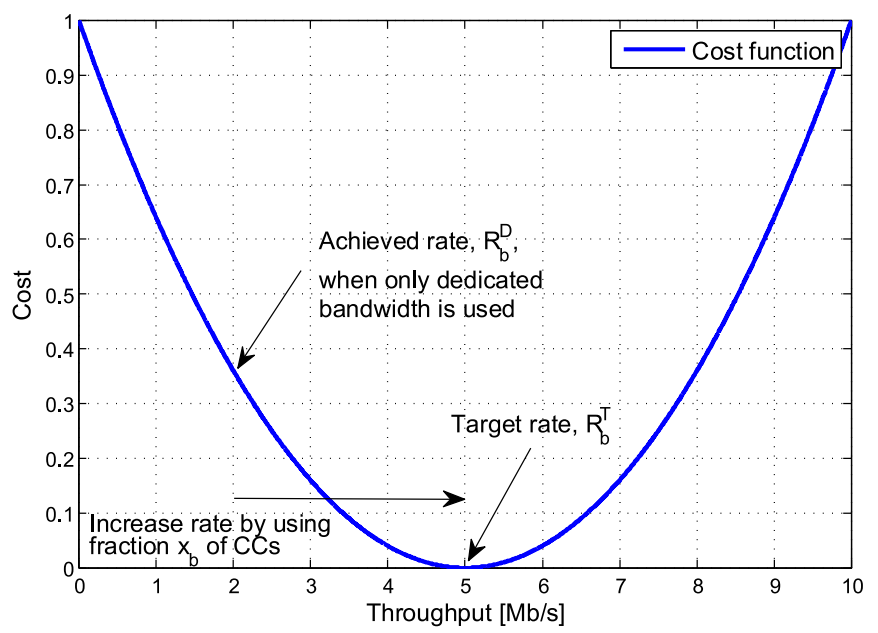

Fig. 3. Example of the cost function shape.

are obtained by simulating a predefined number of channel samples.

\section{Co-Primary Spectrum Sharing Algorithms}

We consider a CoPSS-enabled system where each MNO has its own dedicated bandwidth and has access to a shared common pool of CCs as shown in Fig. 2. The main goal is to satisfy a target rate per SBS $b$, which is achieved by minimizing a time average cost function defined as follows:

$$
\overline{f(t)}=\frac{1}{t} \sum_{\tau=1}^{t}\left(\frac{R_{b}^{\mathrm{D}}(\tau)+x_{b}(\tau) R_{b}^{\mathrm{cc}}(\tau)}{R_{b}^{\mathrm{T}}}-1\right)^{2},
$$

while ensuring a fairness of spectrum sharing among MNOs. However, due to the interference in the network, minimizing the time average cost function of a system with insufficient common bandwidth does not guarantee that the target rate is achieved. Here, $R_{b}^{\mathrm{D}}(\tau)$ is the achievable rate of SBS $b$ when the dedicated bandwidth is used, $x_{b}(\tau)$ is the fraction of CCs used from the common pool by SBS $b$, and $R_{b}^{\text {cc }}(\tau)$ is the summation of the bits that can be transmitted over all the CCs in the common pool. $R_{b}^{\mathrm{T}}$ is the users sum rate target when served by SBS $b$.

Fig. 3 illustrates an example of the cost function shape. In this example, the target rate of a SBS is $5 \mathrm{Mb} / \mathrm{s}$ while the SBS is achieving a rate of $2 \mathrm{Mb} / \mathrm{s}$ using only dedicated bandwidth and the fraction $x_{b}$ of CCs used from the common pool is zero. As the fraction $x_{b}$ increases, the cost function decreases and reaches zero when the achieved throughput is equal to $R_{b}^{T}$. Further increments in $x_{b}$ result higher costs. Thus, the cost is minimized when target throughput is achieved.

The cost minimization problem of SBS $b$ is formally defined as follows:

$$
\begin{aligned}
& \underset{\left(x_{b}(t), \forall t\right)}{\operatorname{minimize}} \overline{f(t)} \\
& \text { subject to } \\
& \left.\quad \frac{1}{T} \sum_{\tau=t-T}^{t} x_{b}(\tau)-v_{\max }\right) \leq \Delta v, \\
& \\
& 0 \leq x_{b}(t) \leq 1,
\end{aligned}
$$


where parameter $v_{\max }$ is the pre-agreed maximum spectrum sharing ratio among operators in which a moving time average of the spectrum usage over time window $T$ which allows MNOs to deviate by $\Delta v$ amount from $v_{\max }$. This constraint is used to guarantee long term fairness between SBSs $/$ MNOs. $^{1}$

\subsection{Learning Algorithm}

The purpose of the proposed approach is to enable SBSs to autonomously select a suitable set of CCs, $x_{b}(t)$, to minimize their cost functions in a decentralized manner. Variable $x_{b}(t)$ is referred to as the action hereinafter. However, the achievable throughput of a SBS depends not only on its own choice of action but also on the remaining SBSs due to the interference caused on the shared CCs. To address this problem we use a reinforcement learning mechanism based on Gibbs sampling [29]. This learning algorithm provides a mechanism to choose $x_{b}(t)$ at each time $t$ with a given probability that depends on the estimated average rate. Moreover, the long term fairness constraint depends on all the variables $x_{b}(t-T), \ldots, x_{b}(t-1)$, which cannot be controlled at the current time instance. Therefore, we modify (2) by moving the fairness constraint as a regularization term in the cost function as follows:

$$
\begin{array}{cl}
\underset{\left(x_{b}(t), \forall t\right)}{\operatorname{minimize}} & \overline{f(t)}+\alpha\left(\bar{v}_{b}(t)-v_{\max }\right) \\
\text { subject to } & 0 \leq x_{b}(t) \leq 1,
\end{array}
$$

where $\bar{v}_{b}(t)=\frac{1}{T} \sum_{\tau=t-T}^{t} x_{b}(\tau)$ is the moving time average of the spectrum usage over time window $T$ and $\alpha \geq 0$ is the regularization coefficient of the spectrum violation.

To solve (3), we propose a learning based algorithm, where SBS $b$ selects a suitable fraction of CCs, $x_{b}(t)$ which maximizes its utility $u_{b}(t)=-\left(\overline{f(t)}+\alpha\left(\bar{v}_{b}(t)-v_{\max }\right)\right)$. Let $\pi_{b}(t)=\left[\pi_{b, 1}(t), \ldots, \pi_{b,\left|\mathcal{A}_{b}\right|}(t)\right]$ be a probability distribution in which SBS $b$ selects its $i$ th action as $x_{b}(t)$ from its action space $\mathcal{A}_{b}=\left\{0, \frac{1}{N_{\mathrm{cc}}}, \ldots, 1\right\}$ at time instant $t$ with $\pi_{b, i}(t)=$ $\operatorname{Pr}\left(x_{b}(t)=\frac{i-1}{N_{\mathrm{cc}}}\right)$, where $i \in\left\{1, \ldots, N_{\mathrm{cc}}+1\right\}$.

For each action SBS $b$ estimates its utility $\hat{u}_{b}(t)=$ $\left[\hat{u}_{b, 1}(t), \ldots, \hat{u}_{b,\left|\mathcal{A}_{b}\right|}(t)\right]$, which is the expected utility for each action over the time period $\{1, \ldots, t-1\}$. At each time $t$, SBS $b$ updates its mixed strategy probability distribution $\pi_{b}(t)$ in which the actions with higher probabilities are exploited while exploring the actions with low probabilities. Such behavior can be captured by the Boltzmann-Gibbs (BG) distribution $G_{b}=\left(G_{b, 1}, \ldots, G_{b,\left|\mathcal{A}_{b}\right|}\right)$ which is calculated as follows:

$$
G_{b, i}\left(\hat{\boldsymbol{u}}_{b}(t)\right)=\frac{\exp \left(\kappa_{b} \max \left(0, \hat{u}_{b, i}(t)\right)\right)}{\sum_{\forall i^{\prime} \in \mathcal{A}_{b}} \exp \left(\kappa_{b} \max \left(0, \hat{u}_{b, i^{\prime}}(t)\right)\right)}, i \in \mathcal{A}_{b},
$$

where $\kappa_{b}>0$ is a temperature parameter that balances between exploration and exploitation. For each time $t$, the joint utility and probability distribution estimations for any SBS $b, \hat{u}_{b}(t)$ and $\pi_{b}(t)$, are updated as follows;

1. Parameter $T$ has to be selected by MNOs, i.e., from minutes to days. In the simulations, $T$ is a fraction of the simulation time. Different time windows are analyzed in the Section 4.

$$
\left\{\begin{aligned}
\hat{u}_{b, i}(t)= & \hat{u}_{b, i}(t-1) \\
& +\gamma_{b}(t) \mathbb{1}_{\left\{x_{b, i}=x_{b}(t-1)\right\}}\left(u_{b}(t)-\hat{u}_{b, i}(t-1)\right), \\
\pi_{b, i}(t)= & \pi_{b, i}(t-1) \\
& +\varepsilon_{b}(t)\left(G_{b, i}\left(\hat{u}_{b}(t-1)\right)-\pi_{b, i}(t-1)\right),
\end{aligned}\right.
$$

with the learning rates satisfying $\lim _{t \rightarrow \infty} \sum_{n=1}^{t} \xi(n)=\infty$ and $\lim _{t \rightarrow \infty} \sum_{n=1}^{t} \xi^{2}(n)<\infty$ for all $\xi=\left\{\gamma_{b}, \varepsilon_{b}\right\}$. The relation between learning rates is such that $\lim _{t \rightarrow \infty} \frac{\gamma_{b}(t)}{\varepsilon_{b}(t)}=0$. Our choice of learning rates are $\gamma_{b}(t)=t^{-\gamma}$ and $\varepsilon_{b}(t)=t^{-\varepsilon}$, with $0.5<$ $\gamma<\varepsilon<1$. Here, $x_{b, i}=\frac{i-1}{N_{\mathrm{cc}}}$ and the operator $\mathbb{1}_{\left\{x_{b, i}=x_{b}(t-1)\right\}}$ returns 1 if $x_{b, i}=x_{b}(t-1)$ is satisfied and 0 otherwise.

The proposed Gibbs algorithm is summarized in Algorithm 1. At the beginning of each time instant $t$, each SBS selects its action $x_{b}(t)$ based on its mixed strategy probability $\pi_{b}(t-1)$, i.e., $x_{b}(t)$ is a random variable with probability mass function $\pi_{b}(t-1)$. Then, SBS picks an appropriate set of CCs that maximizes capacity while minimizing interference from nearby SBSs by relying on the channel quality indicator information, which is an estimation of the quality of the CC. Here, with CQI information, SBSs sort the CCs in an order from highest-to-lowest quality. This procedure allows each SBS to model the capacity as a function of number of CCs. The number of required CCs is derived from how many bits one CC can transfer on average, which gives an accurate estimate. The proposed algorithms based on Gibbs sampling use this knowledge, capacity as a function of number of CCs, to determine the preferred action which maximizes the capacity while ensuring long term fairness among operators. Thus, based on the action $x_{b}$, SBS $b$ selects the best $\left\lceil x_{b} N_{C C}\right\rceil$ from the sorted CCs from the common pool. This procedure eventually minimizes the interference resulting in increased rates as the algorithms converge to stationary distributions.

Algorithm 1. Co-Primary Spectrum Sharing Algorithm Using Gibbs Sampling

1: Each SBS $b$ initializes its probability distribution $\pi_{b}(t)$ and initial utility estimations $\hat{u}_{b}(t)$.

2: while $t>0$ do

3: $\quad$ Each SBS $b$ selects its action $x_{b}(t)$ based on its mixed strategy probability $\pi_{b}(t-1)$.

4: $\quad$ Calculate objective function (3) at time $t$.

5: $\quad$ Update utility estimation and mixed strategy probability using (5).

6: $\quad t \rightarrow t+1$

7: end while

\subsection{Convergence and Optimality Discussion}

Next, we prove that the system of coupled equations in (5) is a Gibbs field with steady-state distribution by invoking the Gibbs-Markov equivalence [30].

Theorem 1. Let $\boldsymbol{a}(t)=\left(a_{1}(t), \ldots, a_{|\mathcal{B}|}(t)\right) \in \mathcal{A}$ be the vector of actions played by all of the SBSs following their mixed strategy probabilities, where $\mathcal{A}=\mathcal{A}_{1} \times \cdots \times \mathcal{A}_{|\mathcal{B}|}$. Let $\pi(t)=\left(\pi_{1}(t), \ldots\right.$, $\left.\pi_{|\mathcal{A}|}(t)\right)$ with $\pi_{i}(t)=\operatorname{Pr}\left(\boldsymbol{a}(t)=\boldsymbol{a}_{i}\right)$ be the probability of selecting ith action $a_{i} \in \mathcal{A}$. Under Algorithm 1, as $t \rightarrow \infty, \pi(t)$ 
converges to a stationary distribution $\Pi=\left(\Pi_{a}, a \forall \in \mathcal{A}\right)$ with,

$$
\Pi_{\boldsymbol{a}}=\frac{\exp \left(\kappa \hat{u}_{\boldsymbol{a}}\right)}{\sum_{\forall \boldsymbol{a}^{\prime} \in \mathcal{A}} \exp \left(\kappa \hat{u}_{\boldsymbol{a}^{\prime}}\right)},
$$

where $\hat{u}_{\boldsymbol{a}}$ is the ensemble average of the action $\boldsymbol{a}^{\prime}$ s utility estimation as $t \rightarrow \infty$.

\section{Proof. See Appendix A.}

Note that for a fixed $\kappa$, the algorithm converges to the stationary distribution $\Pi$ as shown in Theorem 1, and thus the temperature parameter $\kappa$ affects the optimality of the solution as the algorithm converges to $\Pi$. Therefore, we define $\Pi^{(\kappa)}$ as the solution given the value of $\kappa$. In the following, we show that as $\kappa$ approaches infinity, the solution of the proposed algorithm converges to the global solution of the problem (3).

Theorem 2. As $\kappa \rightarrow \infty$,

$$
\lim _{\kappa \rightarrow \infty} \Pi_{a}^{(\kappa)}=\Pi_{a}^{(\infty)}= \begin{cases}\frac{1}{\left|\mathcal{A}^{*}\right|} & \text { if } a \in \mathcal{A}^{*}, \\ 0 & \text { if } a \notin \mathcal{A}^{*}\end{cases}
$$

where $\mathcal{A}^{*}$ is the set of global optimal solutions of (3).

Proof. See Appendix B.

Theorem 2 shows that under a finite $\kappa$ a non-optimal solution can be selected with non-zero probability. Thus, a finite $\kappa$ cannot ensure an optimal selection of actions under the stationary distribution. However, the following theorem shows that the optimality of the solution increases with increasing $\kappa$.

Theorem 3. For any optimal action $a \in \mathcal{A}^{*}$, the probability of selecting the optimal solution after convergence, $\Pi_{a}^{(\kappa)}$, monotonically increases with $\kappa$.

Proof. See Appendix C.

From Theorems 2 and 3, we note that the choice of $\kappa$ affects the optimality of the solution as the algorithm converges to the stationary distribution. Next, we prove that selecting large $\kappa$ decreases the expected cost of the system while increasing the probability of picking the optimal action.

Theorem 4. At convergence, $\Pi^{(\kappa)}$, the expected value of the system utility $E_{\Pi^{(\kappa)}}\left[\hat{u}_{\boldsymbol{a}}\right]$ monotonically increases with $\kappa$.

Proof. See Appendix D.

Theorem 5 (Rate of Convergence). Let $\Pi(t)=\pi_{0} \Omega^{t}$ and $\Pi=\pi_{0} \Omega^{\infty}$ be the probability distribution after the th update and the stationary distribution for a given $\kappa$, respectively, where $\pi_{0}$ is the initial probability distribution, $\Omega$ is the transition matrix of the Markov chain corresponding to the proposed algorithm, and $\Omega^{\infty}$ is the limit of $\Omega^{t}$ as $t \rightarrow \infty$. The proposed algorithm converges to the stationary distribution $\Pi$ with

$$
|| \Pi(t)-\Pi||_{\text {var }} \leq c\left|\lambda_{2}\right|^{t},
$$

where || $\boldsymbol{\Pi}(t)-\boldsymbol{\Pi}\left|\|_{\mathrm{var}}=\frac{1}{2} \sum_{\boldsymbol{a} \in \mathcal{A}}\right| \boldsymbol{\Pi}(t, \boldsymbol{a})-\boldsymbol{\Pi}(\boldsymbol{a}) \mid$ is defined as the total variation distance between two probability distributions $\Pi(t)$ and $\Pi, c$ is a constant with respect to the Markov chain transition matrix $\Omega$, and $\lambda_{2}$ is the second largest eigenvalue of matrix $\Omega$ satisfying $0<\left|\lambda_{2}\right|<1$.

Proof. See Appendix E.

Based on the above theorems, the choice of large $\kappa$ ensures the close global optimality. However, a large $\kappa$ leads Algorithm 1 to exploit a single action from the beginning $(t=1)$ and thus, exploring the rest of the actions is avoided. Therefore, $\kappa$ needs to be bounded for practical implementations.

\subsection{Gibbs with Distribution Update Initialization}

In the original Gibbs algorithm, the uniform distribution over the action space is used as the initialization distribution $\boldsymbol{\pi}_{(0)}$, which is the conventional strategy when Gibbs algorithm is applied [31]. Here, we propose a non-uniform initial distribution in order to improve the performance of the proposed learning algorithm.

The main idea is to modify the initial distribution, which is possible with accurate CQI estimation. Specifically, each SBS estimates the number of CCs needed at the initialization such that

$$
\tilde{x}_{b}=\frac{R_{b}^{\mathrm{T}}-R_{b}^{\mathrm{D}}}{\overline{R_{b}^{\mathrm{cc}}}},
$$

where $R_{b}^{\mathrm{T}}$ is the target rate, $R_{b}^{\mathrm{D}}$ is the achieved rate by using the dedicated bandwidth and $\overline{R_{b}^{\mathrm{cc}}}$ is the mean value of the bits that can be transmitted over all the CCs in the common pool. When each SBS evaluates $\tilde{x}_{b}$, this information is used to calculate the modified initial distribution

$$
\tilde{\pi}_{b, i}=\frac{\tilde{x}_{b}^{i}}{i !} e^{-\tilde{x}_{b}},
$$

which follows the Poisson probability mass function (pmf). However, the Poisson pmf is defined for an infinite number of discrete actions whereas the action space of SBS $b$ is limited by finite choices. Therefore, a truncated Poisson pmf is needed. Here, we truncate the distribution by adding the tail of the original distribution to the most convenient action $\tilde{x}_{b}$. Modifying the uniform distribution improves the learning speed since each SBS already has some insight of which actions should be exploited. However, these SBSs still have opportunity to explore the actions with low probabilities. Fig. 4 shows an example of an initial distribution after estimating how many CCs are needed. In this example the preferred number of CCs is $\tilde{x}_{b}=3$.

\subsection{Proposed Algorithms and Performance Metrics}

We propose three decentralized algorithms (Gibbs, Gibbs+ penalty and Gibbs+distribution) for CoPSS and compare them with three state-of-the-art algorithms (No sharing, Greedy [32] and Equal). The proposed decentralized algorithms do not require sharing any information among MNOs/SBSs. Thus, the proposed CoPSS algorithms are suitable for practical implementations. The baseline models and the proposed algorithms are as follows:

- No sharing: A simple state-of-the-art model in which, SBSs/MNOs do not have access to the common pool of CCs. 


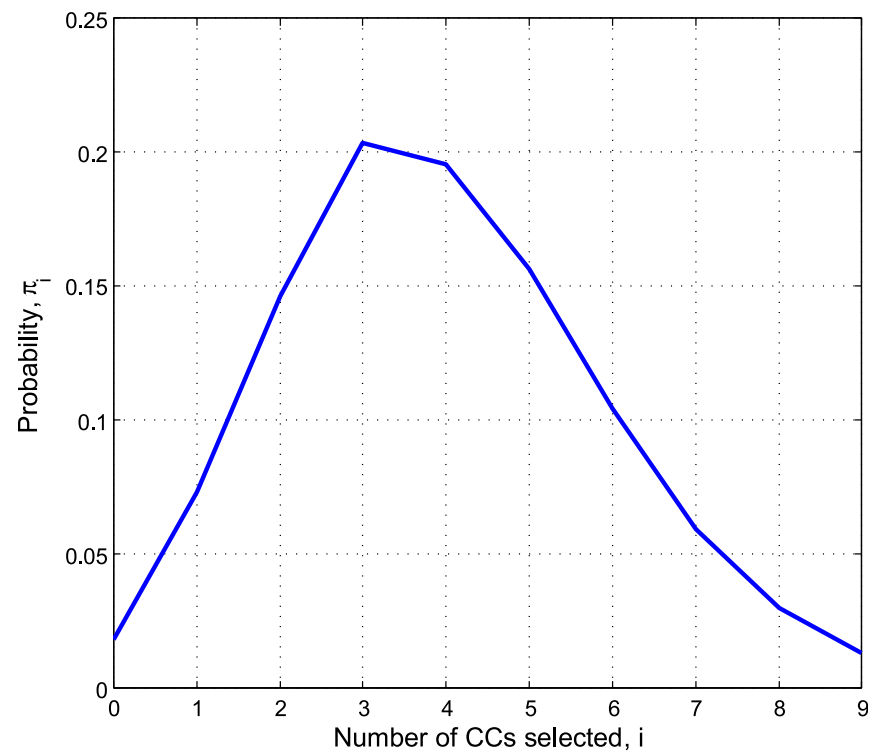

Fig. 4. Example of an initial distribution after estimation.

- Greedy: A decentralized baseline algorithm, where each SBS picks as many CCs as needed in order to achieve the target rate. The greedy approach decides the number CCs to meet the demand, and thus, fairness among operators cannot be guaranteed. When all SBSs adopt the greedy approach, increased interference and decreased rates are expected. This algorithm performs well in scenarios where SBSs are isolated, due to less interference although CCs are shared.

- Equal: A simple state-of-the-art model in which, the common pool of CCs is shared orthogonally and equally between operators. This algorithm performs well in scenarios where SBS are colocated. When SBSs are close to each other, the serving signal and the interference signal have approximately the same strength, resulting to a high FER. Thus, avoiding simultaneous use of the shared CCs is crucial.

- Gibbs: Solving (3) with $\alpha=0$ using learning-based steps (4) and (5) that allows spectrum usage violations in the network.

- Gibbs+penalty: Solving (3) with penalty $(\alpha>0)$ using learning-based steps (4) and (5), which allows instantaneous spectrum usage violations while maintaining long term fairness.

- Gibbs+distribution: Solving (3) with $\alpha=0$ using learning-based steps (4) and (5) that allows spectrum usage violations in the network. The main difference from the conventional Gibbs algorithm is that in the initialization phase, each SBS solves (9) and then (10) is used as its initial distribution, rather than a uniform distribution.

When MNOs are sharing dedicated spectrum, the following information and rules are needed in which MNOs have to agree on the following:

- What is the agreed maximum sharing ratio $v_{\max }$ over the time window (for example equal amount per MNO)? This parameter should be always agreed among MNOs before they start sharing spectrum. The simplest way to define $v_{\max }$ is to divide the bandwidth based on the number of MNOs and set that value as $v_{\max }$.

- Time window length for long term fairness (minutes/hours/days).

- Penalty for exceeding the agreed maximum sharing ratio.

If $\mathrm{MNO}$ change these values without informing others, fairness could not be guaranteed. In this case a central controller is needed that sends this information to each MNO/ SBS. However, this information does not need to be updated frequently and the values could remain the same for days.

The main performance metrics are user throughput and the Jain's index [33], which is a qualitative measure of the fraction of satisfied users. Based on the achieved throughputs, which are normalized by the target rates, the Jain's index for the network is given by

$$
J=\frac{\left(\sum_{b \in \mathcal{B}} \bar{r}_{b}\right)^{2}}{|\mathcal{B}| \sum_{b \in \mathcal{B}} \bar{r}_{b}^{2}}
$$

where $\bar{r}_{b}=\frac{R_{b}^{\mathrm{D}}(\tau)+x_{b}(\tau) R_{b}^{c c}(\tau)}{R_{b}^{\mathrm{T}}}$ is the normalized achieved rate per SBS $b$. The fairest case is when all users achieve their target rates, i.e., Jain's index equals to one. Due to the fact that the normalized achieved rates are used to calculate Jain's index, (11) provides the fairness in terms of satisfying the demands rather the utilization of spectrum. This is to evaluate the performances in terms of QoS. On the contrary, if the fairness is measured in terms of spectrum sharing, no sharing approach becomes the best scheme although it fails to meet the demands.

\section{System Level Based Performance Results}

System level simulations are particularly useful for studying network related issues, such as resource allocation, interference management and mobility management. In this work, a multi-operator Long Term Evolution Advanced (LTE-A) system level simulator is used to model an indoor cellular network, which consist of multiple operators and multiple SBSs per operator.

The simulator uses a layout that has a building of size $120 \mathrm{~m} \times 120 \mathrm{~m}$. The building has one open corridor across it and in total 20 rooms, size $24 \mathrm{~m} \times 24 \mathrm{~m}$ as shown in Fig. 5, which can model an office environment, a shopping mall, an apartment, etc. Internal wall attenuation is $5 \mathrm{~dB}$ per wall [26]. When SBSs are randomly distributed, the number of SBSs in the building is based on deployment probability, e.g., the probability that one room has an SBS. In this layout, users are located at a maximum of $20 \mathrm{~m}$ from the SBS. Users are evenly distributed and each of them is connected to the own MNO's SBS. Moreover, a single user is connected to each SBS. ${ }^{2}$

Results are averaged out over 500 random network topologies, e.g., simulations cover sparse and dense deployments of SBSs/MNOs in the building. Table 1 summarizes the main

2. Parameter $R_{b}^{\mathrm{T}}$ in (1) takes into account the demand of users served by SBS $b$. Therefore, the scenario with multiple users per SBS is equivalent to a scenario with single user per SBS with high demand. Thus, the proposed spectrum sharing algorithm is applicable for multi-user scenarios. The assumption of single user per SBS is simply to reduce simulation time and complexity. 


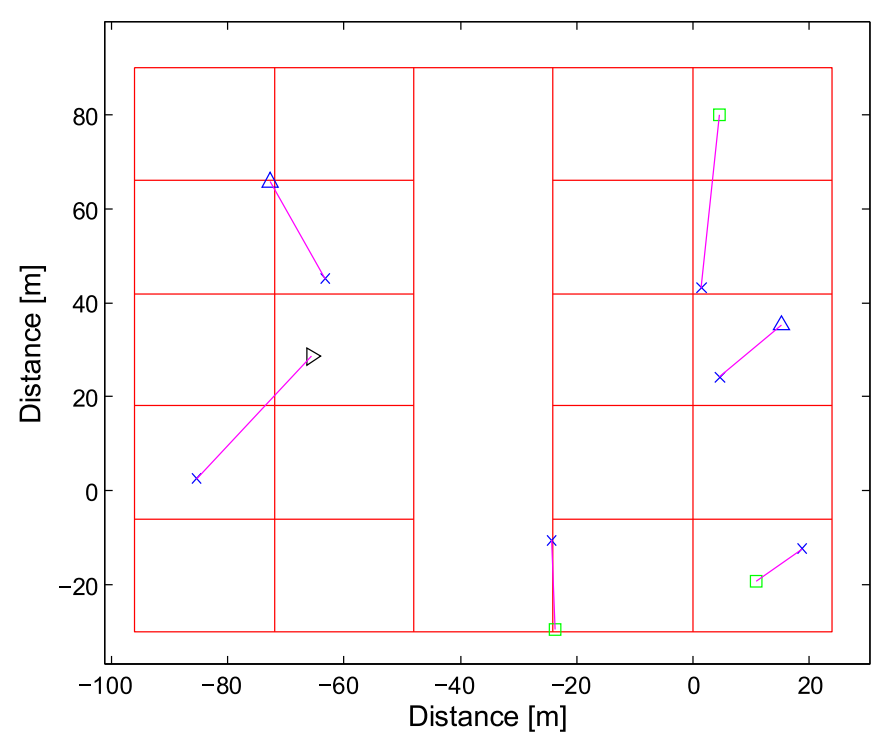

Fig. 5. Small cell layout where base stations are located randomly.

simulation parameters and assumptions used through simulations. Traffic in the network is constant. Thus, delay does not have a big impact on the performance due to the consistency of SBS resource allocation throughout simulations.

In the simulations, continuous constant rate transmission is used. We increase MNO1 target throughput while $\mathrm{MNO} 2$ and MNO3 have a constant target throughput of 5 $\mathrm{Mb} / \mathrm{s}$. This allows us to analyze how different algorithms work when MNOs have similar or different traffic patterns. As already mentioned, in [17], we focused on the possible gains in the achieved throughput when MNOs have similar traffic patterns.

\subsection{Parameter Analysis}

As mentioned in Section 3.1, $\kappa_{b}>0$ is a temperature parameter that balances between exploration and exploitation. Fig. 6

TABLE 1

Simulator Parameters and Assumptions

\begin{tabular}{ll}
\hline Parameterm & \multicolumn{1}{c}{ Assumption } \\
\hline Duplex mode & FDD \\
System bandwidth & $20 \mathrm{MHz}$ \\
Number of CCs & 2 per MNO \\
Number of CCs common pool & 9 \\
Number of users & 1 user per SBS \\
Antenna configurations & $1 \mathrm{Tx}, 2 \mathrm{Rx}$ \\
Receivers & MRC \\
HARQ & Chase combining \\
SBS transmission power & $20 \mathrm{dBm}$ \\
Feedback CQI period & $6 \mathrm{~ms}$ \\
Feedback CQI delay & $2 \mathrm{~ms}$ \\
Traffic model & Continuous constant \\
& rate transmission \\
Internal wall attenuation & $5 \mathrm{~dB}$ \\
Deployment probability & $0.4 \sim 10 \mathrm{SBSs}$ on average \\
Number of MNOs & 3 \\
Carrier frequency & $2 \mathrm{GHz}$ \\
Simulation time & $1,000 \mathrm{~ms}$ \\
Time window $T$ & $300 \mathrm{~ms}$ \\
Penalty coefficient $\alpha$ & 4 \\
Temperature $\kappa_{b}$ & 10 \\
Learning rate exponents $\gamma$ and $\varepsilon$ & 0.6 and 0.95 \\
\hline
\end{tabular}
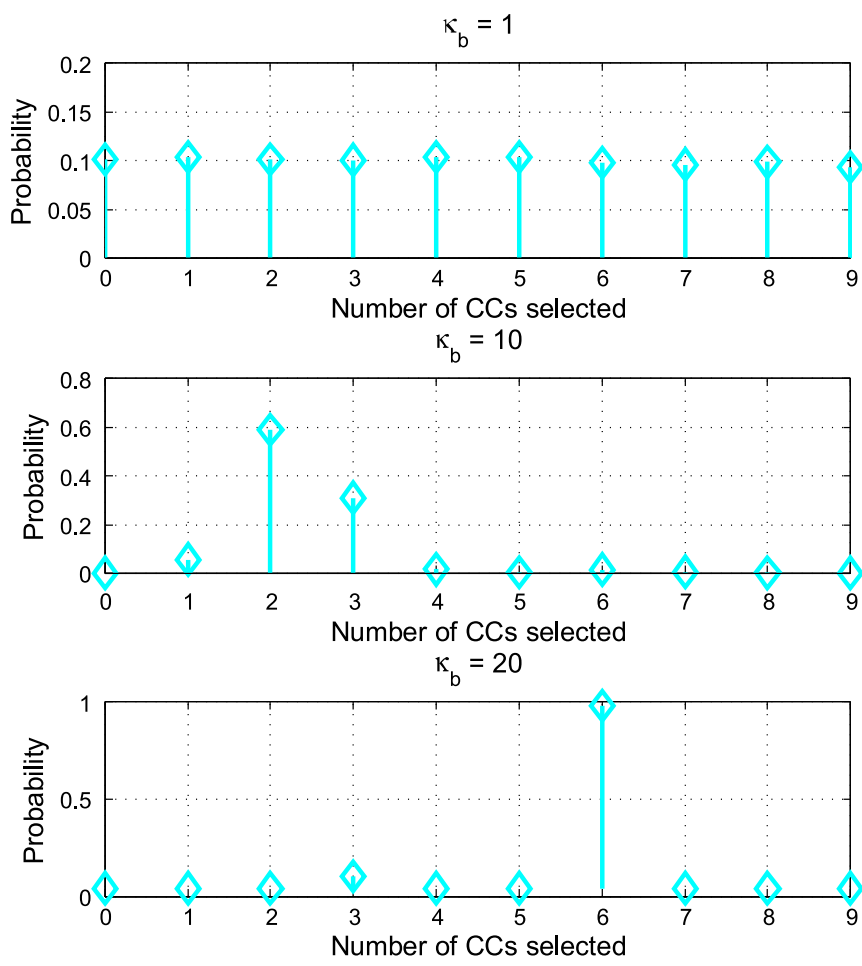

Fig. 6. Final distribution of the Gibbs algorithm with different $\kappa_{b}$ values.

illustrates the impact of the temperature on the resultant final distribution for three different temperature values. When $\kappa_{b}=1$, the final distribution is almost uniform, which means that the temperature is too low and the algorithm does not have enough time to learn how many CCs are needed. When $\kappa_{b}=20$, then the learning is too fast since the probability to select six CCs is almost equal to one (even though the best amount of CCs could be even zero or nine). When $\kappa_{b}=10$, this balances the exploration and exploitation, i.e., efficient learning, and the final distribution is neither uniform as with $\kappa_{b}=1$ nor deterministic as with $\kappa_{b}=20$. The Gibbs+penalty algorithm includes two parameters which control its performance, the time window $T$ and the regularization coefficient of the spectrum violation $\alpha$, which we will refer to as operator's penalty coefficient hereafter. First, we analyze what is the impact of the time window $T$. In Fig. 7a, we show the mean throughput when time window is increased when simulation time is one second. In this scenario, MNO1 target throughput is $20 \mathrm{Mb} / \mathrm{s}$. When the time window is increased higher mean throughput is achieved. The reason is that when time windows are larger, MNOs have more time to violate the pre-agreed maximum sharing ratio. Based on these results, hereinafter we use a time window of $T=300 \mathrm{~ms}$. This allows MNOs to violate for a short period. However, as the simulation time exceeds $T$, SBSs start to limit the spectrum usage if they are violating the pre-agreed maximum sharing ratio. By controlling the time window, higher long term fairness is achieved between MNOs.

The second parameter $\alpha$, is a constant value that controls how significant is the impact of the penalty coefficient on the utility function. In Fig. $7 \mathrm{~b}$, we can see how the achieved mean throughput decreases when the penalty coefficient is increased. For the rest of the simulations $\alpha=4$ is used as a penalty constant that drops the mean throughput by $0.8 \mathrm{Mb} / \mathrm{s}$ when compared to the conventional Gibbs 


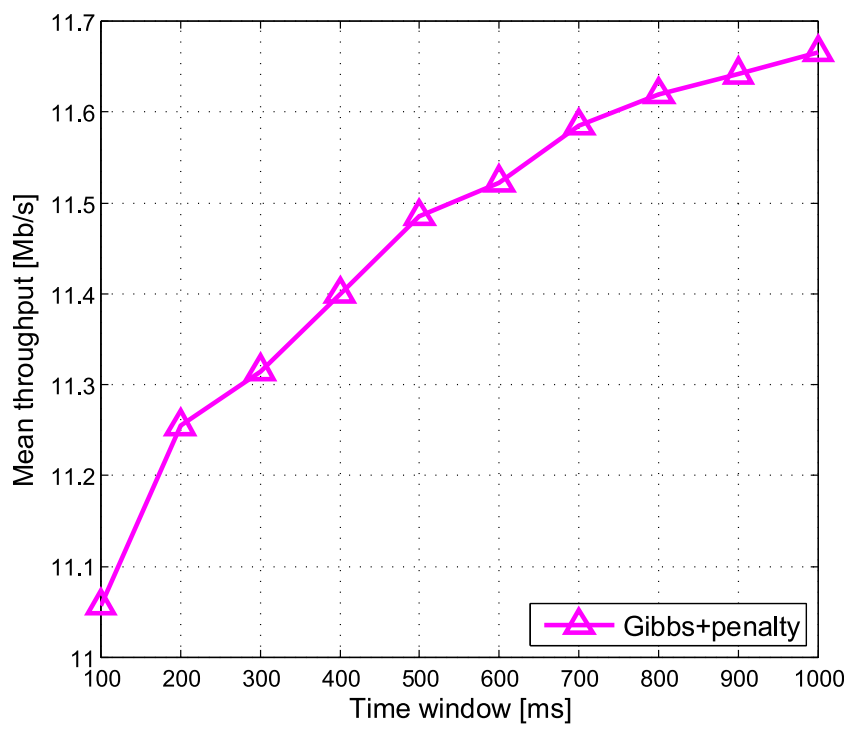

(a) Impact of time window.

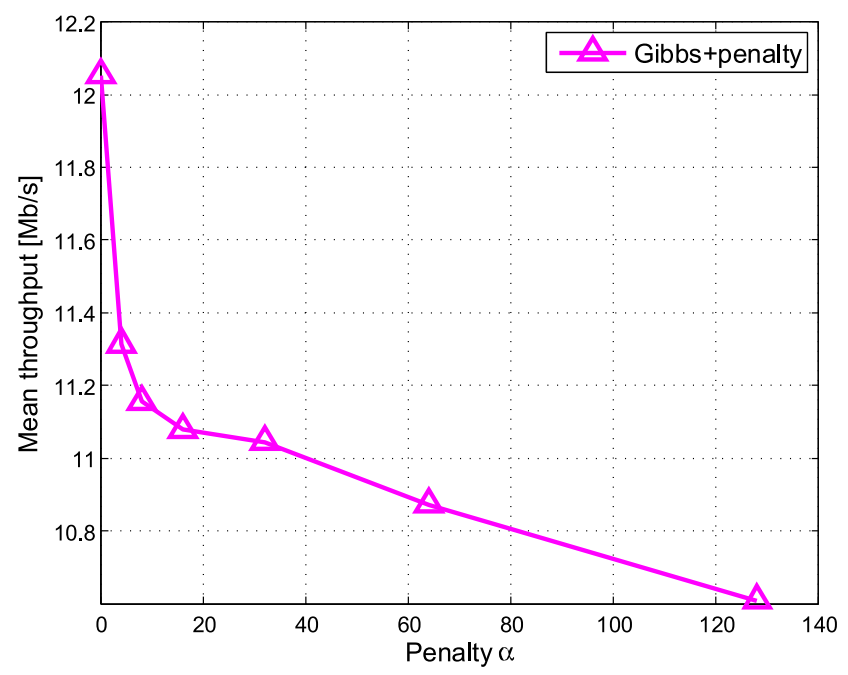

(b) Impact of the operator's penalty coefficient.

Fig. 7. Parameters impact for the mean throughput for the Gibbs+ penalty algorithm.

algorithm $(\alpha=0)$. Our choices of time window $T=300$ and penalty coefficient $\alpha=4$ ensure a sufficiently large penalty term in which the proposed algorithms exhibit considerable performance differences over the conventional Gibbs algorithm.

\subsection{Convergence Analysis}

Fig. 8 compares the convergence speed and the achieved utility between the conventional Gibbs, Gibbs+penalty and Gibbs+distribution algorithms. For all algorithms the convergence speed is similar. However, the achieved utility is higher with the Gibbs+distribution algorithm because the modification of the initial distribution improves the learning procedure. Thus, each SBS already has some insight regarding which actions should be exploited even at the initial phase compared to the conventional Gibbs algorithm. However, the learning curve of the Gibbs+penalty algorithm exhibits a different behavior over the other two methods due to the impact of the penalty term. During the first $100 \mathrm{~ms}$, MNOs exploit the spectrum usage to improve their throughputs by violating

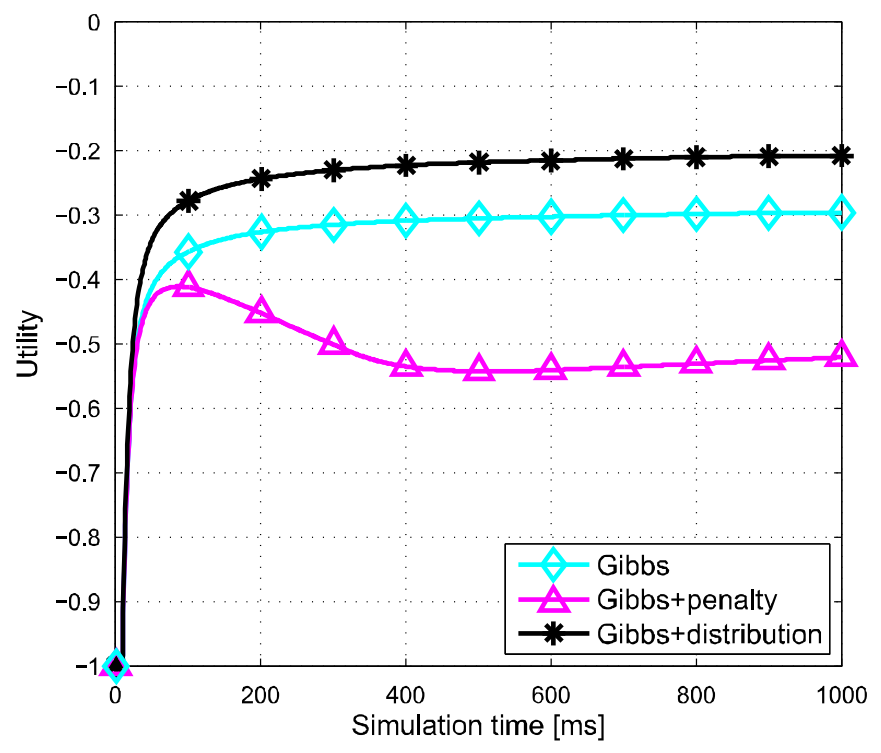

Fig. 8. Convergence speed.

the spectrum sharing ratio. As the simulation time reaches the duration of the time window, these violations result in large penalties and thus, lower utilities. Therefore, MNOs have to compensate the spectrum usage violation, which lowers the throughputs. As the simulation proceeds, MNOs discover the balance between increasing throughputs and maintaining the spectrum usage fairness in which the convergence is achieved. Furthermore, the Gibbs+penalty algorithm will get closer to Gibbs as $\alpha$ goes to zero. In the special case $\alpha=0$ they become equal.

\subsection{Mean Achieved Rates}

The different CoPSS algorithms are analyzed by comparing the mean achieved rates. Fig. 9a shows the achieved mean throughput of MNO1 for different target rates. Here, we can capture how the CoPSS works when MNOs have different throughput targets. In the simulations, we increase MNO1 target throughput while $\mathrm{MNO} 2$ and $\mathrm{MNO} 3$ have a constant target throughput of $5 \mathrm{Mb} / \mathrm{s}$. Note that, for the No sharing case, the throughput saturates around $5 \mathrm{Mb} / \mathrm{s}$ although the demand increases when $\mathrm{MNO}$ cannot access the common pool of CCs. When the demand is low (5-15 $\mathrm{Mb} / \mathrm{s}$ ) the best achieved mean throughput is obtained with the different Gibbs and Equal algorithms while the Greedy provides the worst performance. When the demand increases $(20-30 \mathrm{Mb} / \mathrm{s})$, the Greedy algorithm provides a higher performance while the Equal algorithm starts to saturate. Furthermore, the Gibbs+distribution algorithm provides the overall best performance.

Fig. $9 \mathrm{~b}$ shows the achieved mean throughput of MNO2\&MNO3 for different target rates of MNO1. Again, the No sharing is still the worst, but also the Greedy algorithm exhibits a lower performance compared to the Equal and the Gibbs algorithms. For MNO2\&MNO3, the best algorithm is the Gibbs+penalty algorithm because it is limiting the amount of CCs used by MNO1, which provides more free resources for $\mathrm{MNO} 2 \& \mathrm{MNO}$. The Gibbs+distribution algorithm is very close to the mean performance of the Gibbs+penalty algorithm. All the proposed Gibbs algorithms perform better than the Greedy or the Equal algorithm for MNO2\&MNO3. 


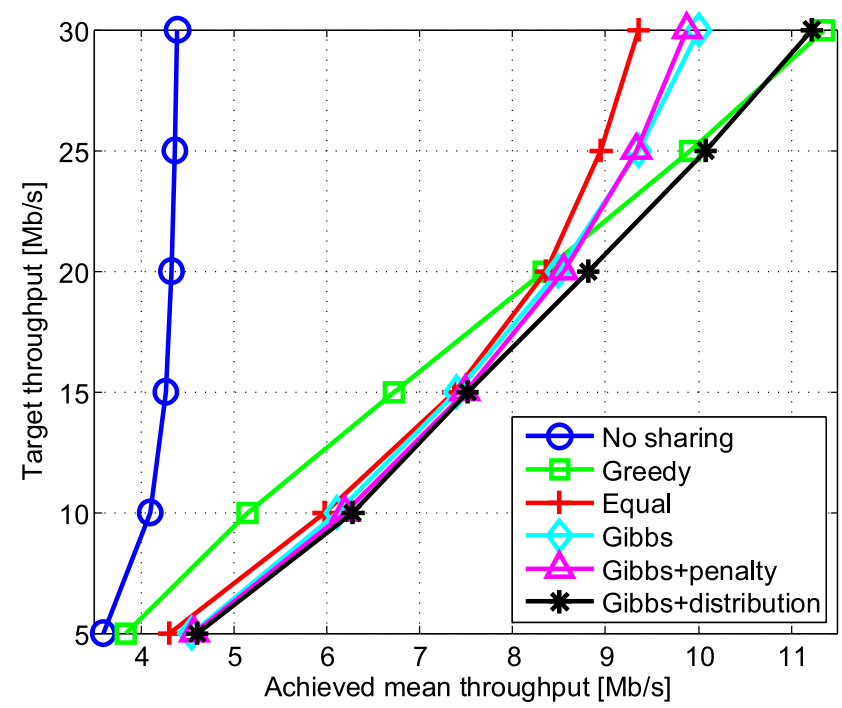

(a) Comparison of the different CoPSS algorithms for MNO1 when downlink target throughput for MNO1 is increased.

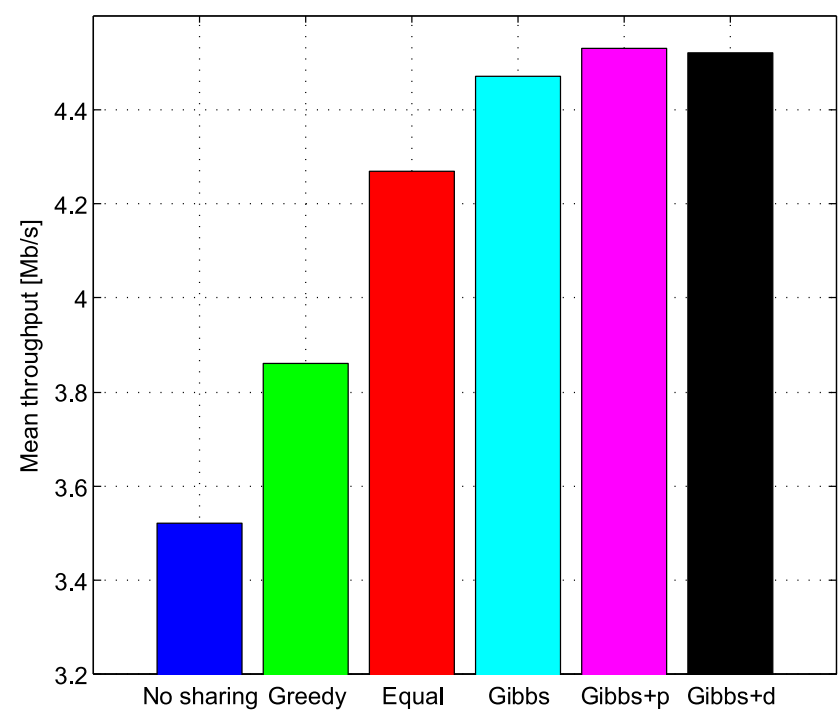

(b) Comparison of the different CoPSS algorithms for MNO2\&MNO3 when downlink target throughput is 5 $\mathrm{Mb} / \mathrm{s}$.

Fig. 9. Performance evaluation of MNOs mean throughput when the downlink target throughput is increased.

\subsection{Performance Analysis for Different Target Rates and Cell Edge}

In Fig. 10, the cumulative distribution function (CDF) of the throughput is analyzed when all MNOs have the same throughput target of $5 \mathrm{Mb} / \mathrm{s}$. The worst performances are achieved with the No sharing and the Greedy algorithms, and all the proposed Gibbs algorithms outperform the the Equal algorithm. The best performance is achieved with the Gibbs +distribution algorithm. When the cell edge (5 percent from $\mathrm{CDFs}$ ) performance is analyzed, all the proposed algorithms outperform state-of-the-art algorithms. Especially, the Gibbs+ distribution offers almost $2.5 \mathrm{Mb} / \mathrm{s}$ better cell edge performance when compared with state-of-art baselines that are in outage. Table 2 summarizes the achievable cell edge users (5 percent from $\mathrm{CDFs}$ ) throughputs for the different algorithms.

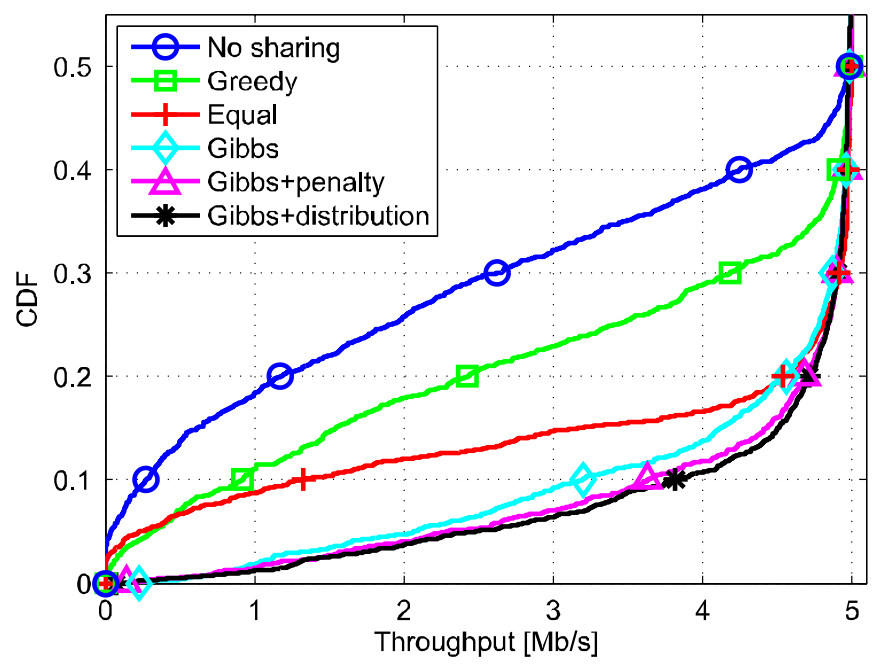

Fig. 10. CDF of MNO1\&MNO2\&MNO3 downlink user throughput for different CoPSS algorithms when the target rate is $5 \mathrm{Mb} / \mathrm{s}$.

Figs. 11a and 11b show the CDF of throughput for MNO1 with $15 \mathrm{Mb} / \mathrm{s}$ target rate and MNO2\&MNO3 with $5 \mathrm{Mb} / \mathrm{s}$ target rate, respectively. For MNO1 the proposed Gibbs algorithms outperform state-of-the-art algorithms. Moreover, throughputs for the cell edge can be vastly improved. The Gibbs+penalty provides $2 \mathrm{Mb} / \mathrm{s}$ while the Greedy and the Equal are in outage. For MNO2\&MNO3, Fig. 11b shows similar performance as in Fig. 10 but now the performance of the Greedy algorithm is even worse than in the previous case. Furthermore, the performance of the Gibbs algorithms is still in acceptable level although there is a slight loss compared to the Equal algorithm, and the cell edge throughput is much higher compared to the Equal algorithm. Table 3 summarizes the achievable cell edge users' (5 percent from CDFs) throughputs for the different algorithms.

In Fig. 12a, we further increase MNO1 throughput demand to $30 \mathrm{Mb} / \mathrm{s}$. Here, the Greedy provides the highest throughput, but still the cell edge is in outage. It can be seen that the Equal algorithm saturates at around $18 \mathrm{Mb} / \mathrm{s}$, whereas the Gibbs and the Gibbs+penalty perform similar to the Equal algorithm from 4 to $18 \mathrm{Mb} / \mathrm{s}$. However, they can provide higher throughput for 30 percent of the users unlike the Equal algorithm. When throughput demand is high, it is clear how the Gibbs+penalty algorithm is limiting the performance compared to the conventional Gibbs algorithm. When the conventional Gibbs achieves the target rate, the Gibbs+penalty provides $26.5 \mathrm{Mb} / \mathrm{s}$ as seen in Fig. 12a. In this scenario, the Gibbs+distribution achieves performance that is close to the Greedy algorithm while guaranteeing $2 \mathrm{Mb} / \mathrm{s}$ higher cell edge performance. As seen in Fig. 12a, further increasing MNO1 throughput demand results performance degrade of $\mathrm{MNO} \& \mathrm{MNO} 3$ that is close to the No sharing, when the Greedy algorithm is used.

TABLE 2

Cell Edge User Throughput [Mb/s] for MNO1\&MNO2\&MNO3 When the Target Rate is $5 \mathrm{Mb} / \mathrm{s}$

\begin{tabular}{lccccc}
\hline No sharing & Greedy & Equal & Gibbs & Gibbs $+\mathrm{p}$ & Gibbs $+\mathrm{d}$ \\
\hline 0.03 & 0.31 & 0.22 & 2.06 & 2.29 & 2.47 \\
\hline
\end{tabular}




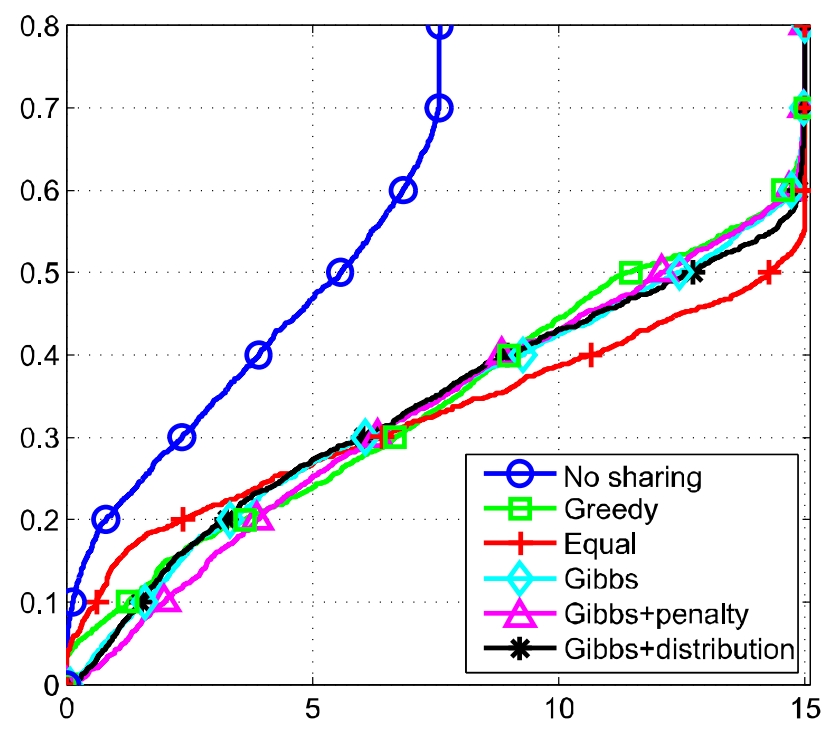

(a) CDF of MNO1 downlink user throughput for different CoPSS algorithms when the target rate is $15 \mathrm{Mb} / \mathrm{s}$.

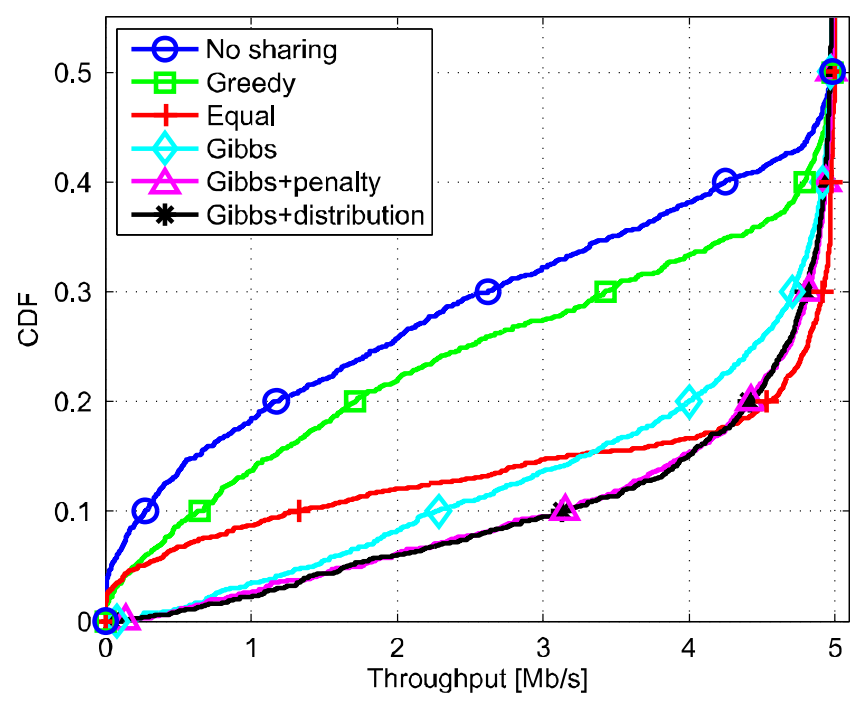

(b) CDF of MNO2\&MNO3 downlink user throughput for different CoPSS algorithms when the target rate is 5 $\mathrm{Mb} / \mathrm{s}$.

Fig. 11. CDF of MNO1 and MNO2\&MNO3 downlink user throughput for different CoPSS algorithms when the target rate is 15 and $5 \mathrm{Mb} / \mathrm{s}$, respectively.

Here, the Gibbs+penalty algorithm provides the best cell edge and overall performance while the Gibbs and the Gibbs+distribution algorithms achieve slightly lower throughput, which is around $0.3 \mathrm{Mb} / \mathrm{s}$. Table 4 summarizes the achievable cell edge users (5 percent from CDFs) throughputs for the different algorithms.

TABLE 3

Cell Edge User Throughput [Mb/s] for MNO1 and MNO2\&MNO3 When the Target Rate is 15 and $5 \mathrm{Mb} / \mathrm{s}$, Respectively

\begin{tabular}{lccccc}
\hline No sharing & Greedy & Equal & Gibbs & Gibbs+p & Gibbs $+\mathrm{d}$ \\
\hline 0 & 0.22 & 0.09 & 0.77 & 1.18 & 0.86 \\
0.03 & 0.20 & 0.22 & 1.43 & 1.75 & 1.67 \\
\hline
\end{tabular}

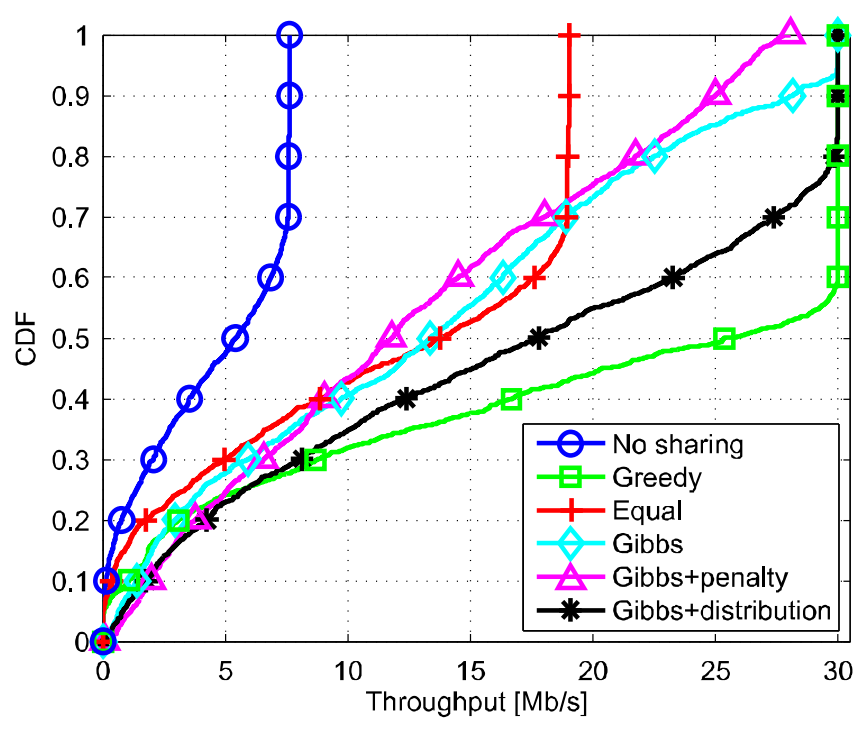

(a) CDF of MNO1 downlink user throughput for different CoPSS algorithms when the target rate is $30 \mathrm{Mb} / \mathrm{s}$.

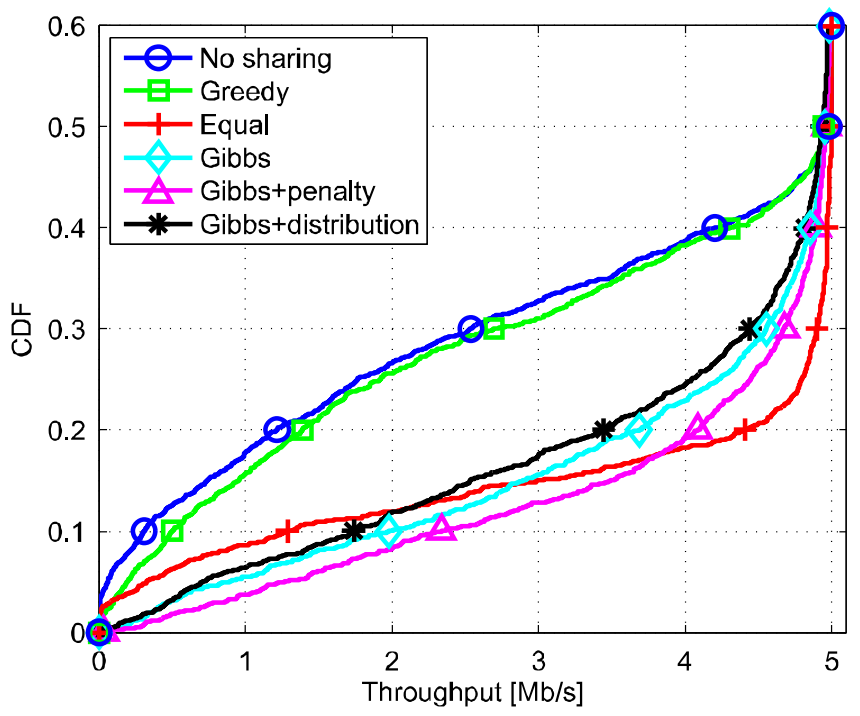

(b) $\mathrm{CDF}$ of $\mathrm{MNO} 2 \& \mathrm{MNO} 3$ downlink user throughput for different CoPSS algorithms when the target rate is 5 $\mathrm{Mb} / \mathrm{s}$.

Fig. 12. CDF of MNO1 and MNO2\&MNO3 downlink user throughput for different CoPSS algorithms when the target rate is 30 and $5 \mathrm{Mb} / \mathrm{s}$, respectively.

\subsection{Fairness of CoPSS}

Finally, in Fig. 13, we present the Jain's index of the whole network for different MNO1 demands and fixed $5 \mathrm{Mb} / \mathrm{s}$ for MNO2\&MNO3. Fig. 13 shows that the Gibbs+ distribution algorithm provides the highest fairness while the Gibbs+penalty is very close to it. All the proposed Gibbs algorithms are outperforming all of state-of-the-art baselines.

TABLE 4

Cell Edge User Throughput [Mb/s] for MNO1 and MNO2\&MNO3 When the Target Rate is 30 and $5 \mathrm{Mb} / \mathrm{s}$, Respectively

\begin{tabular}{lccccc}
\hline No sharing & Greedy & Equal & Gibbs & Gibbs $+\mathrm{p}$ & Gibbs $+\mathrm{d}$ \\
\hline 0.01 & 0.04 & 0.02 & 0.66 & 1.17 & 0.83 \\
0.06 & 0.19 & 0.33 & 0.88 & 1.27 & 0.71 \\
\hline
\end{tabular}




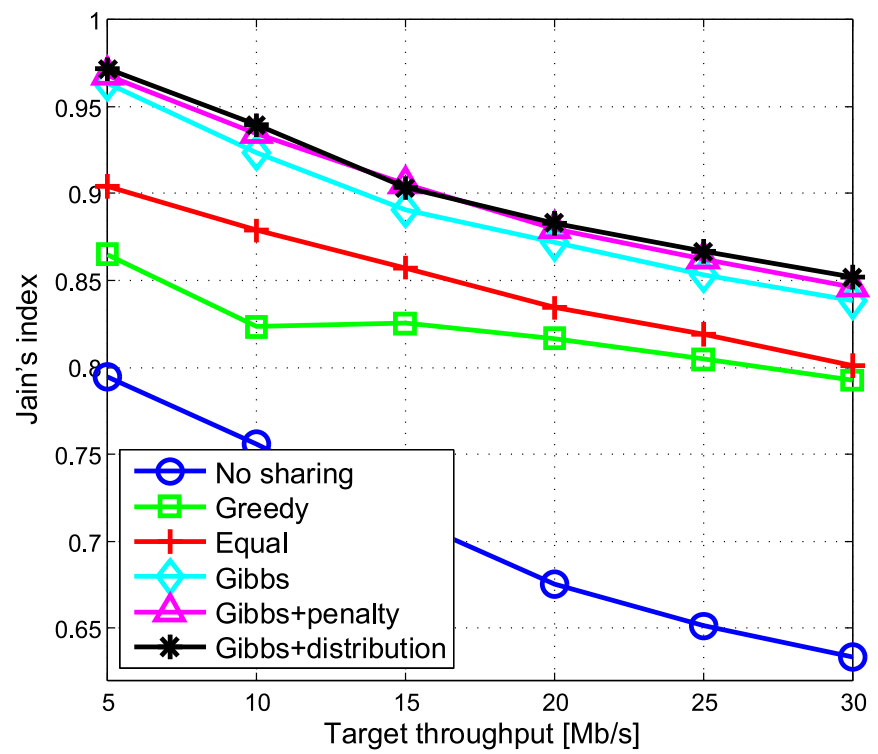

Fig. 13. Jain's index in the random network layout.

Analyzing all the results together, we can conclude that the Gibbs+distribution provides the best performance over state-of-the-art baselines. The proposed Gibbs+distribution algorithm provides higher utility, fairness and throughput. However, the Gibbs+penalty algorithm is suitable for scenarios, where MNOs need more control. Especially over cell edge user throughputs. Although, the proposed algorithms do not require continuous coordination between MNOs, still some level of coordination is needed. For instance, MNOs have to agree what is the pre-agreed maximum sharing ratio and the time window/penalty. However, the required amount of signaling for that is small and thus, the overhead is negligible.

\section{CONCLUSION}

In this article, we have evaluated six different approaches, three state-of-the art methods and three new algorithms for co-primary multi-operator spectrum sharing in a small cell indoor environment. The framework has been established under the LTE-A compliant system simulation platform where the system throughput performance and fairness have been rigorously assessed. Numerical results confirm the potential that co-primary spectrum sharing increases system throughput in the multi-operator setting when mobile network operators share a common pool of component carriers. The results show that learning based algorithms without any coordination between small cell base stations can be used to efficiently share spectrum. The proposed algorithms are suitable for operators with heterogeneous traffic requirements. Especially, the proposed algorithms significantly improve the cell edge performance. Moreover, when high cell-edge performance is required Gibbs+penalty provides the best performance. When the target is to maximize the overall performance, Gibbs+distribution algorithm provides the best performance. Finally, conventional Gibbs algorithm balances the performance between Gibbs+distribution and Gibbs+penalty algorithms in some scenarios. It can be concluded that these proposed algorithms outperform state-of-the-art baselines in all the scenarios. This paper is a foundation for further studies. In our future work, we will consider the impact of coordination among mobile network operators. Furthermore, we will investigate spectrum sharing at higher frequency bands, i.e., $\mathrm{cm} / \mathrm{mm}$-waves.

\section{APPENDIX}

\section{A. Proof of Theorem 1}

Let $u_{b}$ and $u=\sum_{\forall b \in \mathcal{B}} u_{b}$ be the ensemble averages of the utilities of SBS $b$ and the network, respectively. As $t \rightarrow \infty$, for SBS $b$, the ensemble averages of utility estimation, and mixed-strategy probability become $\hat{u}_{b}$ and $\Pi_{b, i}=G_{b, i}\left(\hat{u}_{b}\right)$ for $i \in \mathcal{A}_{b}$ as per (5), respectively. Since $\sum_{b \in \mathcal{B}} \hat{u}_{b, a_{b}}=\sum_{b \in \mathcal{B}} \hat{u}_{b}-$ $u_{b}=\hat{u}_{a}-u=\hat{u}_{\boldsymbol{a}}$ for any action $\boldsymbol{a} \in \mathcal{A}, \hat{u}=\left(\hat{u}_{\boldsymbol{a}}, \forall \boldsymbol{a} \in \mathcal{A}\right)$ is the ensemble average of the utility estimations for the entire system. Since an action $a$ of the system is composed by the set of actions $\left(a_{b, i_{b}}, \forall b \in \mathcal{B}\right)$ per each SBS, the probability of selecting $a$ is $\prod_{\forall b \in \mathcal{B}} \prod_{b, i_{b}}=\prod_{\forall b \in \mathcal{B}} G_{b, i_{b}}\left(\hat{u}_{b}\right)$, which can be simplified as $\Pi_{a}$ in (6).

Following the notation in [30, Chapter 7] we define a Gibbs field with a set of cliques $\mathcal{B}$, a configuration space with a finite size $\mathcal{V}=\prod_{\forall b \in \mathcal{B}} \mathcal{V}_{b}$, a set of finite potentials $\left\{\hat{\boldsymbol{u}}_{b}\left(v_{k}\right), \forall v_{k} \in \mathcal{V}_{b}\right\}_{\forall b \in \mathcal{B}}$, and a probability distribution $\Pi=$ $\left(\Pi_{v}, \forall v \in \mathcal{V}\right)$, where $\Pi_{v}=\exp (\kappa \hat{u}(v)) / \sum_{\forall v^{\prime} \in \mathcal{V}} \exp \left(\kappa \hat{u}\left(v^{\prime}\right)\right)$ and $\hat{u}(v)=\sum_{b \in \mathcal{B}} \hat{u}_{b}\left(v_{k}\right)$ with $v=\left(v_{1}, \ldots, v_{|\mathcal{B}|}\right)$. Suppose that the configuration $v(t)$ at time instant $t$ changes to the configuration $v(t+1)$ at time instant $t+1$ following the probability distribution $\Pi(t)$. Hence, the evolution of the configurations in the above Gibbs field is equivalent to a Markov chain [30, Chapter 7, Theorem 2.1]. The evolution follows a time-inhomogeneous Markov chain as the configuration space $\mathcal{V}$ is finite at any time instant $t$. Since $\Pi(t) \succ 0$ due to the fact that the potentials are finite. Therefore, all configurations have self-loops with positive probability and thus, the Markov chain is aperiodic. Furthermore, since $\Pi(t)$ is a positive vector, $\operatorname{Pr}\left(v(t+1)=v^{\prime \prime} \mid v(t)=v^{\prime}\right)>0$ for any $v^{\prime}, v^{\prime \prime} \in \mathcal{V}$. This verifies that the process starts from configuration $v^{\prime}$ and ends in configuration $v^{\prime \prime}$ with a positive probability, in which the irreducible and positive recurrence properties are held. Since the time-inhomogeneous Markov chain is aperiodic, irreducible and positive recurrent, its transition probability $\Pi$ converges to a stationary distribution as $t \rightarrow \infty[30$, Chapter 7, Theorem 3.1].

It is shown that the transition probability of the above Gibbs field is equivalent to the action selection probability provided by Algorithm 1. Since the transition probability converges to a stationary distribution, the distribution $\Pi$ given in (6) becomes stationary as well.

\section{B. Proof of Theorem 2}

Let $a \in \mathcal{A}^{*}$. Therefore, $\hat{u}_{\boldsymbol{a}}>\hat{u}_{\boldsymbol{a}^{\prime}}$ for all $\boldsymbol{a}^{\prime} \notin \mathcal{A}^{*}$. Then,

$$
\begin{array}{r}
\Pi_{a}^{(\infty)}=\lim _{\kappa \rightarrow \infty} \frac{\exp \left(\kappa \hat{u}_{a}\right)}{\sum_{\forall \boldsymbol{a}^{\prime} \in \mathcal{A}} \exp \left(\kappa \hat{u}_{\boldsymbol{a}^{\prime}}\right)} \\
=\lim _{\kappa \rightarrow \infty} \frac{1}{\left|\mathcal{A}^{*}\right|+\sum_{\forall \boldsymbol{a}^{\prime} \notin \mathcal{A}^{*}} \exp \left(\kappa\left(\hat{u}_{\boldsymbol{a}^{\prime}}-\hat{u}_{\boldsymbol{a}}\right)\right)}=\frac{1}{\left|\mathcal{A}^{*}\right|} .
\end{array}
$$




\section{Proof of Theorem 3}

The first derivative of (6) is

$$
\frac{\partial \Pi_{\boldsymbol{a}}^{(\kappa)}}{\partial \kappa}=\frac{\partial}{\partial \kappa} \frac{\exp \left(\kappa \hat{u}_{\boldsymbol{a}}\right)}{\sum_{\forall \boldsymbol{a}^{\prime} \in \mathcal{A}} \exp \left(\kappa \hat{u}_{\boldsymbol{a}^{\prime}}\right)}=\Pi_{\boldsymbol{a}}\left(\hat{u}_{\boldsymbol{a}^{\prime}}-\mathbb{E}_{\boldsymbol{\Pi}^{(\kappa)}}\left[\hat{u}_{\boldsymbol{a}^{\prime}}\right]\right),
$$

where $\hat{u}=\left(\hat{u}_{\boldsymbol{a}}, \forall \boldsymbol{a} \in \mathcal{A}\right)$ is the utility estimation for the system. Since $\hat{u}_{\boldsymbol{a}} \geq \hat{u}_{\boldsymbol{a}^{\prime}}$ for any $\boldsymbol{a} \in \mathcal{A}^{*}$ and for all $\boldsymbol{a}^{\prime} \in \mathcal{A}$, $\hat{u}_{\boldsymbol{a}^{\prime}}>\mathbb{E}_{\boldsymbol{\Pi}^{(\kappa)}}\left[\hat{u}_{\boldsymbol{a}^{\prime}}\right]$ is true and thus, $\frac{\partial \Pi_{\boldsymbol{a}}^{(\kappa)}}{\partial \kappa}>0$ is held, i.e., the probability of choosing the optimal action monotonically increases with $\kappa$.

\section{Proof of Theorem 4}

Consider the first derivative of the expected utility

$$
\begin{aligned}
\frac{\partial \mathbb{E}_{\boldsymbol{\Pi}^{(\kappa)}}\left[\hat{u}_{\boldsymbol{a}}\right]}{\partial \kappa} & \left.=\frac{\partial}{\partial \kappa} \sum_{\forall \boldsymbol{a} \in \mathcal{A}} \Pi_{\boldsymbol{a}}^{(\kappa)} \hat{u}_{\boldsymbol{a}}\right) \\
& =\sum_{\forall \boldsymbol{a} \in \mathcal{A}} \Pi_{\boldsymbol{a}}^{(\kappa)} \hat{u}_{\boldsymbol{a}}\left(\hat{u}_{\boldsymbol{a}}-\mathbb{E}_{\boldsymbol{\Pi}^{(\kappa)}}\left[\hat{u}_{\boldsymbol{a}}\right]\right) \\
& =\mathbb{E}_{\boldsymbol{\Pi}^{(\kappa)}}\left[\left(\hat{u}_{\boldsymbol{a}}\right)^{2}\right]-\mathbb{E}_{\boldsymbol{\Pi}^{(\kappa)}}^{2}\left[\hat{u}_{\boldsymbol{a}}\right] \\
& =\mathbb{E}_{\boldsymbol{\Pi}^{(\kappa)}}\left[\left(\hat{u}_{\boldsymbol{a}}-\mathbb{E}_{\boldsymbol{\Pi}^{(\kappa)}}^{2}\left[\hat{u}_{\boldsymbol{a}}\right]\right)^{2}\right] .
\end{aligned}
$$

Here, $\mathbb{E}_{\boldsymbol{\Pi}^{(\kappa)}}\left[\left(\hat{u}_{\boldsymbol{a}}-\mathbb{E}_{\boldsymbol{\Pi}^{(\kappa)}}^{2}\left[\hat{u}_{\boldsymbol{a}}\right]\right)^{2}\right]$ is the variance of $\hat{\boldsymbol{u}}$ and $\boldsymbol{\Pi}^{(\kappa)}$, and thus, $\frac{\partial}{\partial \kappa}\left(\mathbb{E}_{\Pi^{(\kappa)}}\left[\hat{u}_{\boldsymbol{a}}\right]\right)>0$ is held.

\section{E. Proof of Theorem 5}

Note that, $\left\|\pi_{0}\right\|_{\mathrm{var}}=\frac{1}{2} \sum_{\boldsymbol{a} \in \mathcal{A}}\left\|\pi_{0}(\boldsymbol{a})\right\|_{1}=\frac{1}{2}$, due to the fact that $\pi_{0}(a) \geq 0$ and $\sum_{\boldsymbol{a} \in \mathcal{A}} \pi_{0}(a)=1$. We have that

$$
\begin{aligned}
\|\Pi(t)-\Pi\|_{\mathrm{var}} & =\left\|\boldsymbol{\pi}_{0} \boldsymbol{\Omega}^{t}-\boldsymbol{\pi}_{0} \boldsymbol{\Omega}^{\infty}\right\|_{\mathrm{var}} \\
& \leq\left\|\boldsymbol{\pi}_{0}\right\|_{\mathrm{var}}\left\|\boldsymbol{\Omega}^{t}-\boldsymbol{\Omega}^{\infty}\right\|_{1}=\frac{1}{4}\left\|\boldsymbol{\Omega}^{t}-\boldsymbol{\Omega}^{\infty}\right\|_{1} .
\end{aligned}
$$

Therefore, to prove Theorem 5, we only need to investigate the properties of $\left\|\Omega^{t}-\Omega^{\infty}\right\|_{1}$. Let $\lambda_{j}$ denote the $j$ th eigenvalue of matrix $\boldsymbol{\Omega}$. We assume that the matrix $\boldsymbol{\Omega}$ has $l$ distinct eigenvalues, satisfying $\left|\lambda_{1}\right|>\left|\lambda_{2}\right| \geq \ldots \geq\left|\lambda_{l}\right|$. Assuming that the algebraic multiplicity of eigenvalue $\lambda_{j}$ is equal to $m_{j}$, we define a $m_{j} \times m_{j}$ matrix $J_{j}$ as follows:

$$
\boldsymbol{J}_{j}=\left[\begin{array}{cccc}
\lambda_{j} & 1 & & 0 \\
& \lambda_{j} & & \\
& & \ddots & 1 \\
0 & & & \lambda_{j}
\end{array}\right]=\lambda_{j} \boldsymbol{I}_{m_{j}}+\underbrace{\left[\begin{array}{cccc}
0 & 1 & & 0 \\
& 0 & & \\
& & \ddots & 1 \\
0 & & & 0
\end{array}\right]}_{\boldsymbol{E}_{j}},
$$

where matrices $I_{m_{j}}$ and $\boldsymbol{E}_{j}$ are the $m_{j} \times m_{j}$ identity matrix and a nilpotent matrix, respectively. Likewise, we define a $\|\mathcal{A}\|_{1} \times m_{j}$ matrix $S_{j}$ such that its $n$th column vector $s_{j, n}$ satisfies $\left(\boldsymbol{\Omega}-\lambda_{j} \boldsymbol{I}_{\|\mathcal{A}\|_{1}}\right)^{n} \boldsymbol{s}_{j, n}=0$. According to the theory of Jordan matrix decomposition [34], the square matrix $\Omega$ can be decomposed into $\Omega=S J S^{-1}$, where $J=\operatorname{diag}\left\{J_{1}\right.$, $\left.J_{2}, \ldots, J_{l}\right\}$ and $S=\operatorname{diag}\left\{S_{1}, S_{2}, \ldots, S_{l}\right\}$. Perron-Frobenius theorem [34] says that, for a positive matrix, there exists a unique largest eigenvalue $\rho$ such that any other eigenvalue is strictly smaller than $\rho$ in absolute value. Meanwhile, only the eigenvalue $\rho$ is associated with a positive eigenvector. Note that $\Omega$ is a positive matrix and has a positive eigenvectors $\Pi$ satisfying $\Pi \Omega=\Pi$. Therefore, the unique largest eigenvalue of matrix $\Omega$ is 1 . That is, $\lambda_{1}=1>\left\|\lambda_{2}\right\|_{1} \geq \ldots \geq\left\|\lambda_{l}\right\|_{1}$. Thus, $J_{1}=[1]$ and $S_{1}$ is a $\|\mathcal{A}\|_{1} \times 1$ matrix.

Taking the $t$ th power of $\Omega$, we have

$$
\begin{gathered}
\boldsymbol{\Omega}^{t}=S\left[\begin{array}{cccc}
1 & 0 & & 0 \\
0 & \boldsymbol{J}_{2}^{t} & & \\
& & \ddots & \\
0 & & & \boldsymbol{J}_{l}^{t}
\end{array}\right] \boldsymbol{\Omega}^{-1} \underset{t \rightarrow \infty}{\rightarrow} \\
\mathbf{\Omega}^{\infty}=S \underbrace{\left[\begin{array}{llll}
0 & 1 & & 0 \\
& 0 & & \\
& & \ddots & 1 \\
0 & & & 0
\end{array}\right]}_{Z} S^{-1} .
\end{gathered}
$$

Since $\left\|\lambda_{j}\right\|_{1}<1$ for $j \geq 2$, we get

$$
\begin{aligned}
\boldsymbol{J}_{j}^{t}= & {\left[\lambda_{j} \boldsymbol{I}_{m_{j}}+\boldsymbol{E}_{j}\right]^{t} } \\
= & \lambda_{j}^{t} \boldsymbol{I}_{m_{j}}+\frac{t \lambda_{j}^{t}}{\lambda_{j}} \boldsymbol{E}_{j}+\ldots \\
& +\frac{t ! \lambda_{j}^{t}}{\left(m_{j}-1\right) !\left(t-m_{j}+1\right) ! \lambda_{j}^{m_{j}-1}} \boldsymbol{E}_{j}^{m_{j}-1} \\
\leq & C \lambda_{j}^{t}\left[\boldsymbol{I}_{m_{j}}+\boldsymbol{E}_{j}+\ldots+\boldsymbol{E}_{j}^{m_{j}-1}\right],
\end{aligned}
$$

with $C>0$. This implies that any $\boldsymbol{J}_{j}^{t}$ satisfies

$$
\boldsymbol{J}_{j}^{t} \leq C \lambda_{2}^{t}\left[\boldsymbol{I}_{m_{j}}+\boldsymbol{E}_{j}+\cdots+\boldsymbol{E}_{j}^{m_{j}-1}\right],
$$

since $\left\|\lambda_{2}\right\|_{1} \geq \cdots \geq\left\|\lambda_{l}\right\|_{1}$. Therefore,

$$
\begin{aligned}
& \left\|\boldsymbol{\Omega}^{t}-\boldsymbol{\Omega}^{\infty}\right\|_{1} \leq\|S\|_{1}\left\|\boldsymbol{J}^{t}-Z\right\|_{1}\left\|S^{-1}\right\|_{1} \\
\leq & \left\|\boldsymbol{I}_{\|\mathcal{A}\|_{1}}\right\|_{1}\left\|\boldsymbol{J}^{t}-Z\right\|_{1} \leq \underbrace{C|\mathcal{A}| \sum_{j=2}^{l} \frac{m_{j}\left(m_{j}+1\right)}{2}}_{c}\left|\lambda_{2}\right|^{t},
\end{aligned}
$$

where matrix $Z$ is denoted in (17). Together with (15), it follows that $\left\|\Pi(t)-\left.\Pi\left|\|_{\mathrm{var}} \leq c\right| \lambda_{2}\right|^{t}\right.$, i.e., (8). This concludes the proof.

\section{ACKNOWLEDGMENTS}

This research was supported by the Finnish Funding Agency for Technology and Innovation (TEKES), Nokia, Anite, Huawei Technologies, and Infotech Oulu Graduate School. Kari Horneman and Ling Yu, from Nokia, earn special thanks for invaluable guidance for this study.

\section{References}

[1] Nokia, "Looking ahead to 5G, white paper,"

[2] "Nokia solutions and networks, optimising spectrum utilisation towards 2020, white paper,"

[3] "METIS deliverable D5.1 Intermediate description of the spectrum needs and usage principles," https://www.metis2020.com/wpcontent/uploads/deliverables/METIS_D5.1_v1.pdf

[4] B. Aazhang, J. Lilleberg, and G. Middleton, "Spectrum sharing in a cellular system," in Proc. IEEE 8th Int. Symp. Spread Spectrum Techn. Appl., Aug. 2004, pp. 355-359. 
[5] M. Bennis and J. Lilleberg, "Inter-operator resource sharing for 3G systems and beyond," in Proc. IEEE 9th Int. Symp. Spread Spectrum Techn. Appl., Aug. 2006, pp. 401-405.

[6] F. Mazzenga, M. Petracca, R. Pomposini, F. Vatalaro, and R. Giuliano, "Performance evaluation of spectrum sharing algorithms in single and multi operator scenarios," in Proc. IEEE 73rd Veh. Technol. Conf., May 2011, pp. 1-5.

[7] L. Anchora, M. Mezzavilla, L. Badia, and M. Zorzi, "A performance evaluation tool for spectrum sharing in multi-operator LTE networks," Comput. Commun., vol. 35, no. 18, pp. 2218-2226, Aug. 2012.

[8] Sharing physical resources mechanisms and implementations for wireless networks (SAPHYRE). (2012). [Online]. Available: http:/ / www.saphyre.eu/index.html

[9] E. Jorswieck, L. Badia, T. Fahldieck, E. Karipidis, and J. Luo, "Spectrum sharing improves the network efficiency for cellular operators," IEEE Commun. Mag., vol. 52, no. 3, pp. 129-136, Mar. 2014.

[10] J. Panchal, R. Yates, and M. Buddhikot, "Mobile network resource sharing options: Performance comparisons," IEEE Trans. Wireless Commun., vol. 12, no. 9, pp. 4470-4482, Sep. 2013.

[11] P. Ahokangas, et al., "Defining co-primary spectrum sharing A new business opportunity for MNOs?" in Proc. 9th Int. Conf. Cognitive Radio Oriented Wireless Netw. Commun., Jun. 2014, pp. 395-400.

[12] Y. Teng, Y. Wang, and K. Horneman, "Co-primary spectrum sharing for denser networks in local area," in Proc. 9th Int. Conf. Cognitive Radio Oriented Wireless Netw. Commun., Jun. 2014, pp. 120-124.

[13] B. Singh, et al., "Coordination protocol for inter-operator spectrum sharing in co-primary 5G small cell networks," IEEE Commun. Mag., vol. 53, no. 7, pp. 34-40, Jul. 2015.

[14] T. Sanguanpuak, N. Rajatheva, and M. Latva-Aho, "Co-primary spectrum sharing with resource allocation in small cell network," in Proc. 1st Int. Conf. 5G Ubiquitous Connectivity, Nov. 2014, pp. 6-10.

[15] I. F. Akyildiz, P. Wang, and S.-C. Lin, "SoftAir: A software defined networking architecture for 5G wireless systems," Comput. Netw., vol. 85, pp. 1-18, 2015.

[16] S.-C. Lin, P. Wang, and M. Luo, "Jointly optimized QoS-aware virtualization and routing in software defined networks," Comput. Netw., vol. 96, pp. 69-78, 2016.

[17] P. Luoto, P. Pirinen, M. Bennis, S. Samarakoon, S. Scott, and M. Latva-Aho, "Co-primary multi-operator resource sharing for small cell networks," IEEE Trans. Wireless Commun., vol. 14, no. 6, pp. 3120-3130, Jun. 2015.

[18] P. Luoto, M. Bennis, P. Pirinen, S. Samarakoon, and M. Latva-Aho, "Gibbs sampling based spectrum sharing for multi-operator small cell networks," in Proc. IEEE Int. Conf. Commun. Workshop, Jun. 2015, pp. 967-972.

[19] L. P. Qian, Y. J. A. Zhang, and M. Chiang, “Distributed nonconvex power control using Gibbs sampling," IEEE Trans. Commun., vol. 60, no. 12, pp. 3886-3898, Dec. 2012.

[20] WINNER II channel models, D1.1.2 V1.2. (2008). [Online]. Available: http:/ / http:/ / www.ist-winner.org/deliverables.html

[21] H. Pennanen, T. Haataja, J. Leinonen, A. Tölli, and M. Latva-Aho, "System level evaluation of TDD based LTE-Advanced MIMOOFDMA systems," in Proc. IEEE GLOBECOM Workshops, Dec. 2010, pp. 809-813.

[22] T. Haataja, H. Pennanen, J. Leinonen, A. Tölli, and M. Latva-Aho, "Space-frequency scheduling in TDD based LTE-advanced MIMO-OFDMA systems," in Proc. IEEE 73rd Veh. Technol. Conf., May 2011, pp. 1-5.

[23] BeFEMTO. (2013). [Online]. Available: http://www.ict-befemto. eu/home.html

[24] P. Luoto, J. Leinonen, P. Pirinen, V. V. Phan, and M. Latva-Aho, "Bitmap based resource partitioning in LTE-A femto deployment," in Proc. IEEE Int. Conf. Commun., Jun. 2013, pp. 5005-5009.

[25] P. Luoto, P. Pirinen, and M. Latva-Aho, "Energy efficient load sharing in LTE-A hetnets," in Proc. IEEE 9th Int. Conf. Wireless Mobile Comput. Netw. Commun., Oct. 2013, pp. 119-123.

[26] "BeFEMTO. Assumptions For System Level Calibration. Version 0.8, Mar. 2011," http://www.ict-befemto.eu/fileadmin/ documents/publications/deliverables/BeFEMTO-D2.1.pdf

[27] 3rd Generation Partnership Project, Technical Specification Group Radio Access Network, Spatial channel model for Multiple Input Multiple Output (MIMO) simulations, 3GPP Standard, Tech. Rep. 25.996 v6.1.0.

[28] X. He, K. Niu, Z. He, and J. Lin, "Link layer abstraction in MIMOOFDM system," in Proc. Int. Workshop Cross Layer Des., Sep. 2007, pp. $41-44$.
[29] L. P. Qian, Y. J. A. Zhang, and M. Chiang, "Distributed nonconvex power control using Gibbs sampling," IEEE Trans. Commun., vol. 60 , no. 12 , pp. 3886-3898, Dec. 2012.

[30] P. Brémaud, Markov Chains: Gibbs Fields Monte Carlo Simulation and Queues. Berlin, Germany: Springer, 1999.

[31] S. Borst, M. Markakis, and I. Saniee, "Nonconcave utility maximization in locally coupled systems, with applications to wireless and wireline networks," IEEE/ACM Trans. Netw., vol. 22, no. 2, pp. 674-687, Apr. 2014.

[32] T. H. Cormen, R. L. Leiserson, C. E. Rivest, and C. Stein, Introduction to Algorithms. Cambridge, MA, USA: MIT Press, 2009.

[33] R. Jain, D. Chiu, and W. Hawe, "A quantitative measure of fairness and discrimination for resource allocation in shared computer systems," CoRR, 1998. [Online]. Available: http://arxiv. org/abs/cs.NI/9809099

[34] A. Berman and R. Plemmons, Nonnegative Matrices in the Mathematical Sciences. Philadelphia, PA, USA: SIAM, 1994. 